\title{
SPHERE RECOGNITION LIES IN NP
}

\author{
SAUL SCHLEIMER
}

\begin{abstract}
We prove that the three-sphere recognition problem lies in NP. Rubinstein [Haifa, 1992] and Thompson [Math. Res. Let., 1994] showed that the problem is decidable. Our result relies on Casson's version [MSRI, 1997] of their algorithm and recent results of Agol, Hass, and Thurston [STOC, 2002].
\end{abstract}

\section{INTRODUCTION}

The three-sphere recognition problem asks: given a triangulation $T$, is the underlying space $|T|$ homeomorphic to the three-sphere? To solve this problem, Rubinstein [23] introduced almost normal two-spheres (see Section 4). Thompson [26] greatly simplified Rubinstein's proof using Gabai's technique of thin position [8].

Theorem 1.1 (Rubinstein [23], Thompson [26]). The three-sphere recognition problem lies in EXPTIME.

Casson [5] then introduced the novel idea of crushing triangulations along normal two-spheres (see Section 13). This reduced the space complexity.

Theorem 14.1 (Casson [5]). The three-sphere recognition problem lies in PSPACE.

Following Casson's algorithm and work of Agol, Hass, and Thurston [2] (see Theorem 4.6) we show the following.

Theorem 15.1. The three-sphere recognition problem lies in NP.

That is, any triangulation $T$ of the three-sphere admits a polynomialsized certificate: a proof that $T$ is indeed a triangulation of the threesphere (see Section 3). Theorem 15.1 has an immediate corollary.

Corollary 1.2. The three-ball recognition problem lies in NP.

Date: September 18, 2011.

This work is in the public domain. 
Proof. Theorem 3.4 gives a polynomial-time algorithm to verify that $|T|$ is a three-manifold. Suppose that $T$ is a triangulation of the three-ball. First verify that that $S=\partial|T|$ is a two-sphere by checking connectedness and Euler characteristic. Next, build $D(T)$ : the triangulation obtained by doubling across $S$. Next, Theorem 15.1 gives a certificate that $|D(T)|$ is a three-sphere. Finally, Alexander's Theorem [11, Theorem 1.1] implies that the two-sphere $S$ bounds a three-ball in $|D(T)|$.

A surface vector $v(S)$ is the vector of normal coordinates of $S$ with respect to the triangulation $T$ (Section 4.1). Here is a result closely related to Corollary 1.2.

Corollary 1.3. The following problem lies in NP: given a triangulation $T$ of a three-manifold and a surface vector $v(S)$, decide if $S$ bounds a three-ball in $|T|$.

Proof. Using Lemma 4.5 to compute the Euler characteristic and using Theorem 4.6 to check connectedness, verify that $S$ is a two-sphere. Crush the triangulation $T$ along the surface $S$ to obtain a triangulation $T^{\prime}$ (Section 13). Theorem 5.9 of Jaco and Rubinstein's paper [16] tells us that the connect sum of the components of $\left|T^{\prime}\right|$ is homeomorphic to $|T|$, up to keeping track of lens space summands.

Following Casson, Barchechat's thesis [3, page 50] gives a polynomialtime algorithm that reassembles these lens spaces and the components of $\left|T^{\prime}\right|$, recovering $|T|$. Thus we only need to check that these lens spaces and the components of $\left|T^{\prime}\right|$, arising as submanifolds of the ball bounded by $S$, are three-spheres. The former are dealt with as in [3]. The latter are certified using Theorem 15.1.

We next state a technical result, involved in the proof of Theorem 15.1, that may be of independent interest.

Theorem 12.1. There is a polynomial-time algorithm that, given a triangulation $T$ of an oriented three-manifold and $v(S)$ where $S$ is a transversely oriented almost normal surface, produces as output $v(\operatorname{norm}(S))$, the vector for the normalization of $S$.

Corollary 1.3, Theorem 12.1, and the bounds given by [25, Chapter 6 ] result in the following.

Corollary 1.4. The following problem lies in NP: given a triangulation $T$ of a closed orientable irreducible atoroidal three-manifold, decide if $|T|$ is a surface bundle over the circle.

Corollary 1.4, unfortunately, is very far from proving that bundle recognition lies in NP; certifying irreducibility or atoroidality are interesting and difficult open questions. Note that certifying zero-efficiency 
would in turn certify irreducibility. Corollary 1.4 is similar to a earlier result of Ivanov [13]. He shows that recognition of the three-sphere, amongst the class of zero-efficient triangulations (there called irreducible $Q$-triangulations), lies in NP.

There are other problems in three-manifold topology lying in NP. Hass, Lagarias, and Pippenger [10] have shown that the unknotting problem, first solved by Haken, lies in NP. Agol [1] has given a proof, using sutured manifold hierarchies, that the recognition of Haken manifolds lies in NP (see also [15]). Agol's algorithm requires, as the base case, some version of Corollary 1.3. Agol deduces that the unknotting problem lies in co-NP. Agol, Hass and Thurston [2] have shown that the 3-manifold knot genus problem is NP-complete. For a discussion of algorithmic three-manifold topology we refer the reader to [10] or to Matveev's book [20].

Historical note. Three-sphere recognition (Theorem 1.1) is a fundamental result in low-dimensional topology. Accordingly, many expositions exist. A solution to the problem was first presented by Rubinstein [23] in a series of lectures in Haifa, Israel, in 1992. Thompson [26] gave a different proof, relying on Gabai's technique of thin position [8] soon after. Rubinstein's papers [24, 22] lay out his original ideas on the problem. Shortly after Thompson's paper appeared, Matveev [19] gave an exposition of her algorithm using handle decompositions instead of triangulations; his paper gives a particularly elegant version of the lightbulb trick, replacing Thompson's "fluorescent light bulb trick" [26, Section 3.1]. Next, Casson [5], in lectures at MSRI, California, introduced the idea of crushing triangulations along normal two-spheres. An exposition of the Rubinstein-Thompson algorithm was given by Ivanov [13], stated in the language of $Q$-triangulations. Barchechat's thesis [3, Chapter 6.1] gives an exposition of Casson's algorithm; it has been implemented by Burton in his computer program Regina [4].

Regarding the present work: the material in Sections 7 to 10 had its genesis as Chapter 4 of my thesis [25], supervised by Andrew Casson; this material has not been otherwise published. Ian Agol, when we were both at the University of Illinois, Chicago, suggested that those techniques might bear on the computational complexity of three-sphere recognition. This paper, first posted to the arXiv in 2004, is the result. Another novelty, also introduced here, is to use the Agol-HassThurston machinery [2] to produce a normalization algorithm that runs in polynomial time (Theorem 12.1).

In 2008, Sergei Ivanov [14] published a different proof of Theorem 15.1. As in this paper, his argument closely follows Casson's algorithm. Our 
polynomial-time normalization is, in his paper, replaced by a discussion of vertex fundamental surfaces (following Hass-Lagarias-Pippinger [10]) together with the intriguing idea of crushing along almost normal two-spheres. It is an interesting question whether his method is more efficient than ours. His paper also shows that the problem of recognizing manifolds with compressible boundary lies in NP.

Acknowledgments. I thank both Andrew Casson and Ian Agol for many enlightening mathematical conversations. I thank the mathematics department at UIC for its support during the writing of this paper. I thank the referees for their comments and corrections.

\section{Sketch of the Proof of the Main theorem}

We closely follow Casson's algorithm [5] for recognizing the threesphere. Fix $T$, a triangulation of $S^{3}$. Produce a certificate $\left\{\left(T_{i}, v\left(S_{i}\right)\right)\right\}_{i=0}^{n}$ as follows: The triangulation $T_{0}$ is equal to $T$. For every $i$, Lemma 4.13 provides $S_{i}$, a normal two-sphere in $T_{i}$ that is not vertex-linking, if such exists. If $T$ is zero-efficient then Lemma 4.13 provides $S_{i}$, an almost normal two-sphere in $T_{i}$. Definitions are given in Section 4.

If $S_{i}$ is normal apply Theorem 13.1: $T_{i+1}$ is obtained from $T_{i}$ by crushing $T_{i}$ along $S_{i}$. Briefly, we cut $\left|T_{i}\right|$ along $S_{i}$, cone the resulting two-sphere boundary components to points, and collapse non-tetrahedral cells of the resulting cell structure to obtain the triangulation $T_{i+1}$. This is discussed in Section 13, below.

If $S_{i}$ is almost normal then obtain $T_{i+1}$ from $T_{i}$ by deleting the component of $\left|T_{i}\right|$ that contains $S_{i}$. Finally, the last triangulation $T_{n}$ is empty, as is $S_{n}$.

That completes the construction of the certificate. We now turn to the procedure for checking a given certificate; we cite a series of polynomial-time algorithms that verify each part of the certificate. Begin by checking that $T$ is a triangulation of a three-manifold that is a homology three-sphere, using Theorems 3.4 and 3.5. Next, check if $T=T_{0}$ using Lemma 3.1. For general $i$, verify that $S_{i}$ is a two-sphere by checking its Euler characteristic (Lemma 4.5) and checking that it is connected (Theorem 4.6). Next, if $S_{i}$ is normal verify that the triangulation $T_{i+1}$ is identical to the triangulation obtained by crushing $T_{i}$ along $S_{i}$. To do this in polynomial time requires Theorem 13.1 and Lemma 3.1. If $S_{i}$ is almost normal then check that the component $T^{\prime}$ of $T_{i}$ containing $S_{i}$ satisfies $\left|T^{\prime}\right| \cong S^{3}$ using Theorems 12.1 and 10.3.

Finally, by Theorem 13.2, for every $i$ we have that $\#\left|T_{i}\right| \cong \#\left|T_{i+1}\right|$ where the connect sum on the left hand side ranges over the components of $\left|T_{i}\right|$ while the right hand side ranges over the components of $\left|T_{i+1}\right|$. By 
definition the empty connect sum is $S^{3}$, and this finishes the verification of the certificate.

\section{Definitions}

Complexity theory. Please consult $[9,21]$ for more thorough treatments.

A problem $P$ is a function from a set of finite binary strings, the instances, to another set of finite binary strings, the answers. If $T$ is an instance we use size $(T)$ to denote the length of $T$. A problem $P$ is a decision problem if the range of $P$ is the set $\{0,1\}$. A solution for $P$ is a Turing machine $\mathcal{M}$ that, given an instance $T$ on its tape, computes and then halts with only the answer $P(T)$ on its tape. We will engage in the usual abuse of calling such a Turing machine an algorithm (or procedure) that solves the problem $P$.

An algorithm $\mathcal{M}$ runs in polynomial time if there is a polynomial $q$ so that, for any instance $T$, the machine $\mathcal{M}$ halts in time at most $q(\operatorname{size}(T))$. Computing $q$ precisely, or even its degree, is a delicate question and sensitive to the exact model of computation. Thus one simply says that a decision problem lies in $\mathbf{P}$ if it has some polynomial-time solution.

A decision problem $P$ lies in NP if there is a polynomial $q$ with the following property: For all instances $T$ with $P(T)=1$ there is a proof of length at most $q(\operatorname{size}(T))$ that $P(T)=1$. Such a polynomial-length proof is a certificate for $T$. More concretely: Suppose that there is a polynomial $q^{\prime}$ and a Turing machine $\mathcal{M}^{\prime}$ so that, for every instance $T$ with $P(T)=1$, there is a string $C$ where $\mathcal{M}^{\prime}$ run on $(T, C)$ outputs the desired proof that $P(T)=1$ in time less than $q^{\prime}(\operatorname{size}(T))$. Then, again, the problem $P$ is in NP and we again call $C$ a certificate for $T$.

A decision problem lies in PSPACE if there is a polynomial $q$ so that on every instance $T$ and for every step of the computation the distance between the first and last non-blank squares of the tape of $\mathcal{M}$ is at most $q(\operatorname{size}(T))$.

A decision problem lies in EXPTIME if there is a polynomial $q$ so that that the Turing machine $\mathcal{M}$ halts in time at most $\exp (q(\operatorname{size}(T)))$ on every instance $T$. Note that $\mathbf{P} \subset \mathbf{N P} \subset \mathbf{P S P A C E} \subset \mathbf{E X P T I M E}$. At least one of these inclusions is strict as $\mathbf{P} \neq \mathbf{E X P T I M E}$.

Triangulations. A model tetrahedron $\tau$ is a copy of the regular Euclidean tetrahedron of side length one with vertices labeled by $0,1,2$, and 3. See Figure 1 for a picture. Label the six edges by their vertices $(0,1),(0,2)$, and so on. Label the four faces by the number of the vertex they do not contain. The standard orientation on $\mathbb{R}^{3}$ induces an 
orientation on the model tetrahedron which in turn induces orientations on the faces.

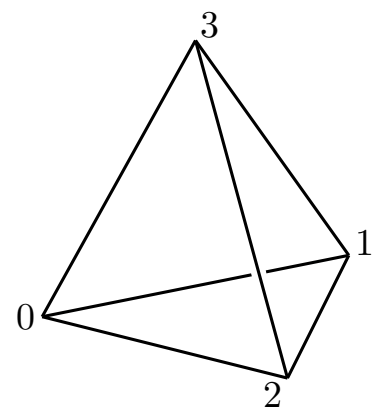

Figure 1. A regular Euclidean tetrahedron with all sidelengths equal to one.

A labeled triangulation $T$, of size $n$, is a collection of $n$ model tetrahedra $\left\{\tau_{i}\right\}_{i=1}^{n}$, each with a unique name, and a collection of face pairings.

Here a face pairing is a triple $(i, j, \sigma)$ specifying a pair of tetrahedra $\tau_{i}$ and $\tau_{j}$ as well as an isometry $\sigma$ from a face of $\tau_{i}$ to a face of $\tau_{j}$. We will omit the labelings when they are clear from the context.

A triangulation is not required to be a simplicial complex. However every face must appear in exactly two face pairings or in none. We do allow face pairings of the form $(i, i, \sigma)$ but, as a matter of convenience, we do not allow a face to be glued to itself.

Lemma 3.1. There is a polynomial-time algorithm that, given triangulations $T$ and $T^{\prime}$, decides whether or not $T$ is identical to $T^{\prime}$.

Proof. Recall that $T$ and $T^{\prime}$ are labeled: all of the tetrahedra come equipped with names. To check for isomorphism check that every name appearing in $T$ also appears in $T^{\prime}$ and that all of the face pairings in $T$ and $T^{\prime}$ agree.

Remark 3.2. Note that, for unlabeled triangulations, there is still a quadratic algorithm that determines isomorphism of triangulations. This is because an isomorphism is determined by the image of a single tetrahedron.

Let $|T|$ be the underlying topological space; the space obtained from the disjoint union of the model tetrahedra by taking the quotient by the face pairings. Notice that $|T|$ is not, in general, a manifold.

At this point we should fix an encoding scheme which translates triangulations into binary strings. However we will not bother to do more than remark that there are schemes which require about $n \log (n)$ 
bits to specify a triangulation with $n$ tetrahedra. (This blow-up in length is due to the necessity of giving the tetrahedra unique names.) Thus we will abuse notation and write $\operatorname{size}(T)=n$ even though the representation of $T$ as a binary string is somewhat longer.

Topology. Recall that the three-sphere is the three-manifold

$$
S^{3}=\left\{x \in \mathbb{R}^{4} \mid\|x\|=1\right\} .
$$

The connect sum $M \# N$ of two connected oriented three-manifolds $M$ and $N$ is obtained by removing an open three-ball from the interior of each of $M$ and $N$ and gluing the resulting two-sphere boundary components with an orientation reversing homeomorphism. The connect sum naturally extends to a collection of connected, oriented threemanifolds; if $M$ is the disjoint union of connected three-manifolds then \# $M$ denotes their connect sum.

Note that Alexander's Theorem [11, Theorem 1.1] implies that $M \# S^{3}$ is homeomorphic to $M$, for any three-manifold $M$. We adopt the convention that the empty connect sum yields the three-sphere.

Definition 3.3. Suppose that $T$ is a triangulation and suppose that $p \in|T|$. Fix $\epsilon$, sufficiently small, and take $\epsilon$-neighborhoods about the preimages of $p$ in the model tetrahedra. Each is a cone on a subsurface of the sphere. These fit together to form a cone on a two-complex $S_{p}$, the link of $p$. If $p \in T^{0}$ then $S_{p}$ is called a vertex link.

We now relate several algorithms which take triangulations and check topological properties. See [10, 17, 20] for in-depth discussions.

Theorem 3.4. There is a polynomial-time algorithm that, given a triangulation $T$, decides whether or not $|T|$ is a three-manifold.

Proof. The underlying space $|T|$ is a quotient of a disjoint union of finitely many model tetrahedra. Furthermore, the face pairings are isometries. It follows that $|T|$ is second-countable and Hausdorff.

It remains only to verify that every point $p \in|T|$ has a neighborhood homeomorphic to a three-ball. Equivalently, every point $p$ has link $S_{p}$ being a sphere or a disk. This is automatic for points lying in the interior of tetrahedra. Since faces cannot be glued to themselves, any point in the interior of a face also has the desired link.

Now suppose that $p$ lies in the interior of an edge. The link $S_{p}$ is a union of spherical lunes. Thus $S_{p}$ is $D^{2}, S^{2}$, or $\mathbb{R P}^{2}$. The latter may happen only at the midpoint of an edge.

Finally, suppose that $p$ is a vertex. Now $S_{p}$ is a union of spherical triangles. Again, $p$ has the desired three-ball neighborhood if and only if $S_{p}$ is a sphere or a disk. 
Thus the algorithm need only check how tetrahedra are glued around an edge and the topology of each vertex link. In terms of size $(T)$ there are at most linearly many edges and vertices. Checking each edge and each vertex link takes at most polynomial time. This is because there are at most $6 \cdot \operatorname{size}(T)$ tetrahedra around any edge. Also, each vertex link is a union of at most $4 \cdot \operatorname{size}(T)$ spherical triangles.

Recall that a three-manifold $M$ is a homology three-sphere if it has the same homology groups as $S^{3}$.

Theorem 3.5. There is a polynomial-time algorithm that, given a triangulation $T$ of a three-manifold, decides whether or not $|T|$ is a homology three-sphere.

Proof. The homology groups $H_{*}(|T|, \mathbb{Z})$ may be read off from the Smith normal forms of the chain boundary maps: we refer the reader to [7, Section 2] for an accessible overview of algorithmic computation of homology. Finally, the Smith normal form of an integer matrix may be computed in polynomial time [12].

We record a few consequences of the homology three-sphere assumption.

Lemma 3.6. If $M^{3}$ is a homology three-sphere then $M$ is connected, closed, and orientable. Every closed, embedded surface in $M$ is orientable and separating. Every connect summand of $M$ is also a homology threesphere.

It follows that if $N$ is a connect summand of a homology three-sphere and $N$ is a lens space then $N \cong S^{3}$.

\section{Normal And almost nORmal SURFACES}

In order to study triangulations we first discuss Haken's theory of normal surfaces. See [10] for a detailed discussion, including references to the foundational work of Haken and Schubert. Other references on normal surfaces include $[16,3]$.

Definition 4.1. An isotopy $H:|T| \times I \rightarrow|T|$ is a normal isotopy if, for all $s \in I$ and for every simplex $\sigma$ in $T, H_{s}(\sigma)=\sigma$.

We make the same definition for faces, model tetrahedra, and subcomplexes of the triangulation. Two subsets of such are normally isotopic if there is a normal isotopy taking one to the other.

For example, suppose that $f$ is a face of a model tetrahedron $\tau$. There are three normal isotopy classes of properly embedded arcs with end points in distinct edges of $f$. Any such arc in $f$ is called a normal arc. 
A simple closed curve $\alpha \subset \partial \tau$ is a normal curve if $\alpha$ is transverse to the one-skeleton of $\tau$ and $\alpha$ is a union of normal arcs. The length of a normal curve $\alpha$ is the number of normal arcs it contains. A normal curve $\alpha$ is called short if it has length three or four.

Lemma 4.2. Suppose that $\alpha \subset \partial \tau$ is a connected normal curve. The following are equivalent:

- $\alpha$ is short.

- $\alpha$ meets every edge of $\tau^{1}$ at most once.

- $\alpha$ misses some edge of $\tau^{1}$.

Proof. To see this, let $\left\{v_{i j} \mid 0 \leq i<j \leq 3\right\}$ be the number of intersections of $\alpha$ with each of the six edges of $\tau$. There are twelve inequalities $v_{01} \leq v_{12}+v_{02}$, and so on. Additionally there are six congruences $v_{01}+v_{12}+v_{02} \equiv 0$, and so on, all modulo two. An easy argument now gives the desired results.

In a model tetrahedron there are seven normal isotopy classes (or types) of normal disk, corresponding to the seven distinct short normal curves in $\partial \tau$. See Figure 2. These are the four normal triangles and three normal quads. The triangles are of type $0,1,2$, or 3 depending on which vertex they cut off of the model tetrahedron, $\tau$. The quads are of type 1, 2, or 3 depending on which vertex is with 0 when $\tau$ is cut by the quad.
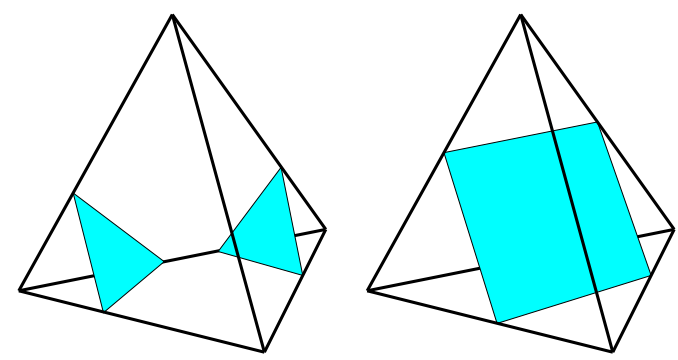

Figure 2. Two of the four triangles and one of the three quads.

Definition 4.3. A surface $S$, properly embedded in $|T|$, is normal if for every model tetrahedron $\tau \in T$ the preimage of $S$ in $\tau$ is a collection of normal disks.

There is also the almost normal octagon and almost normal annulus, defined by Rubinstein [22]. See Figure 3 for examples. An octagon is a disk in the model tetrahedron bounded by a normal curve of length eight. An annulus is obtained by taking two disjoint normal disks and tubing 
them together along an arc parallel to an edge of the model tetrahedron. A surface $S$ properly embedded in $|T|$ is almost normal if the preimage of $S$ in $\tau$ is a collection of normal disks for every tetrahedron $\tau \in T$, except one. In the exceptional tetrahedron there is a collection of normal disks and exactly one almost normal piece.
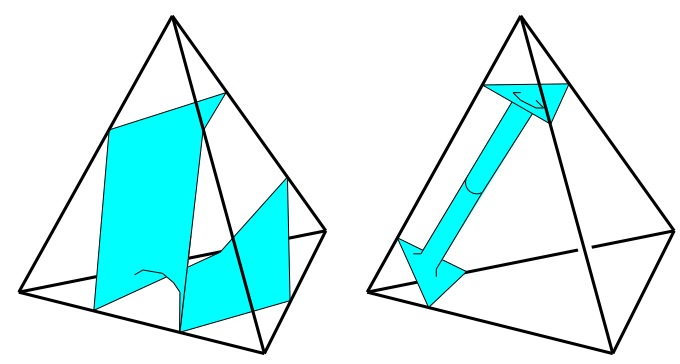

FiguRE 3. One of the three octagons and one of the 25 annuli.

Remark 4.4. Following Jaco and Rubinstein [16, page 74] we do not allow, as an almost normal surface, parallel normal surfaces connected by an almost normal annulus contained in the product region between them. We also remark that in many cases of interest the almost normal annulus can be removed from the theory. For example, see the proof of Proposition 5.12 in [16].

4.1. Weight and Euler characteristic. For any surface $S \subset|T|$, transverse to the skeleta, define its weight to be the number of intersections between $S$ and the one-skeleton $T^{1}$ : weight $(S)=\left|S \cap T^{1}\right|$. We record a normal surface $S$ as a surface vector $v(S) \in \mathbb{Z}^{7 \cdot \operatorname{size}(T)}$. The first $4 \cdot \operatorname{size}(T)$ coordinates describe the number of normal triangles of each type while the last $3 \cdot \operatorname{size}(T)$ coordinates describe the number of normal quads of each type. At least two-thirds of these last $3 \cdot \operatorname{size}(T)$ coordinates are zero as an embedded surface has only one type of normal quad in each tetrahedron.

For an almost normal surface $S$ we again record the vector $v(S)$ of normal disks, as well as the type of the almost normal piece and the name of the tetrahedron containing it. (There is a small issue when the almost normal piece is an annulus obtained by tubing a pair of normal disks of the same type. Then $v(S)$ has length $7 \cdot \operatorname{size}(T)+1$ as one parallel collection of normal disks may be interrupted by the almost normal piece.) Note that two normal (or almost normal) surfaces have the same vector if and only if they are normally isotopic.

We now have a few results concerning normal and almost normal surfaces. We assume throughout that the triangulation $T$ has underlying 
space a three-manifold. We first reproduce Algorithm 9.1 from [17]. See the end of Section 5 in [2] for a similar treatment.

Lemma 4.5. There is a polynomial-time algorithm that, given a triangulation $T$ and a normal or almost normal surface vector $v(S)$, computes the weight of $S$ and the Euler characteristic of $S$.

Proof. To find the weight of $S$ on a single edge $e$ of $T^{1}$ count the number of normal disks meeting $e$ (with multiplicity depending on how many times the containing tetrahedron meets $e$ ) and divide by the valency of $e$ in $T^{2}$, the two-skeleton.

For the Euler characteristic simply use the formula $\chi(S)=F-E+V$ and the cell structure on $S$ coming from its being a normal surface. (If $S$ contains an almost normal annulus then we must add a single edge running between the two boundary components of the annulus.) The number of faces is the sum of the coordinates of $v(S)$. The number of edges is $3 / 2$ times the sum of the triangle coordinates plus twice the sum of the quad coordinates. The number of vertices of $S$ can be computed from $v(S)$ and the degrees of the edges in $T^{1}$. Small corrections are necessary when $S$ is almost normal.

Theorem 4.6 (Agol-Hass-Thurston [2]). There is a polynomial-time algorithm that, given a triangulation $T$ and a normal or almost normal surface vector $v(S)$, produces integers $n_{i}$ and surface vectors $v\left(F_{i}\right)$ so that

- $v(S)=\sum n_{i} \cdot v\left(F_{i}\right)$,

- if $i \neq j$ then $F_{i} \cap F_{j}=\emptyset$, and

- if $i \neq j$ then $v\left(F_{i}\right) \neq v\left(F_{j}\right)$.

Proof. This is one application of the "extended counting algorithm" given in [2]. See the proof of Corollary 17 of that paper.

4.2. Haken sums. Suppose $S, F, G$ are three non-empty normal surfaces with $v(S)=v(F)+v(G)$. Then we say that $F$ and $G$ are compatible: in every tetrahedron where both $F$ or $G$ have quads, these quads are of the same type. After a normal isotopy of $F$ and $G$ we find that $S$ is the Haken sum of $F$ and $G$; there is a cut-and-paste of $F$ and $G$ constructing $S$. It follows that $\chi(S)=\chi(F)+\chi(G)$.

Likewise, suppose $S$ and $F$ are almost normal with identical almost normal piece, $G$ is normal, the quads of $G$ are disjoint from the almost normal piece of $F$, and the normal coordinates add: $v(S)=v(F)+v(G)$. Again we say that $S$ is a Haken sum. If $F$ contains an octagon then we may normally isotope $G$ so that no normal triangle of $G$ meets the octagon. If $F$ contains an annulus we may normally isotope $G$ so that 
no triangle of $G$ meets the annulus in a meridian of the tube. In either case we may perform cut-and-paste and find $\chi(S)=\chi(F)+\chi(G)$.

When $S$ is a Haken sum as above we write $S=F+G$. If $S$ is not a Haken sum then $S$ is fundamental.

Lemma 4.7. If $S=F+G$, where $G$ is a vertex link, then $S$ is not connected.

Lemma 4.8. If $S \subset|T|$ is a fundamental normal or almost normal surface then the largest entry of $v(S)$ is at most $\exp (\operatorname{size}(T))$.

Proof. There is a constant $c$ (not depending on $T$ or $S$ ) such that the largest entry of $v(S)$ is less than $2^{c \cdot \operatorname{size}(T)}$. This lemma is proved for normal surfaces in [10, Lemma 6.1]. In the almost normal case, when the almost normal piece is an octagon, we obtain similar bounds using the system of linear equations provided by [26, Section 5]. The case of the annulus is similar.

Lemma 4.9. Suppose $T$ is a triangulation of a homology three-sphere. Suppose $T$ contains a non-vertex-linking normal two-sphere. Then $T$ contains a fundamental non-vertex-linking normal two-sphere.

Proof. This is similar to work of Haken and Schubert; our statement follows directly from Proposition 5.7 of [16]. The essential points are that Euler characteristic is additive under Haken sum, that $T$ does not contain any normal $\mathbb{R P}^{2}$ or $\mathbb{D}^{2}$ (by Lemma 3.6), and that no summand is vertex-linking (by Lemma 4.7).

Definition 4.10. Fix a triangulation $T$ so that $|T|$ is a three-manifold. If every normal two-sphere is vertex linking then, following Jaco and Rubinstein [16], we say that $T$ is zero-efficient.

Lemma 4.11. Suppose $T$ is a zero-efficient triangulation of a homology three-sphere. Suppose T contains an almost normal two-sphere. Then $T$ contains a fundamental almost normal two-sphere.

Proof. This is identical to the proof of Lemma 4.9, except that $S$ cannot have a normal two-sphere summand as $T$ is zero-efficient.

Of a much different level of difficulty is the following.

Theorem 4.12 (Rubinstein [23], Thompson [26]). If $|T| \cong S^{3}$ then $T$ contains an almost normal two-sphere.

We end this section with a useful lemma.

Lemma 4.13. There is an exponential-time algorithm that, given a triangulation $T$ of a closed three-manifold 
- produces the surface vector of a fundamental non-vertex-linking normal two-sphere or, if none exists,

- produces the surface vector of a fundamental almost normal two-sphere or, if neither exists,

- reports that $|T|$ is not homeomorphic to the three-sphere.

Proof. We only sketch a proof - the interested reader should consult [10], [16, page 66] or [3, page 83]. If $T$ admits a non-vertex-linking normal twosphere or an almost normal two-sphere then, by Lemmas 4.9 and 4.11 there is a fundamental such surface. This surface can now be found by enumerating all fundamental surfaces (a finite list, by work of Haken) and checking Euler characteristics (Lemma 4.5). On the other hand, if no almost normal two-sphere exists then Theorem 4.12 implies that $|T|$ is not the three-sphere.

As presented the running time of the algorithm is unclear. The time depends on the number of fundamental surfaces. However, in both cases (normal or almost normal) fundamental solutions may be replaced by vertex fundamental surfaces $[17,5]$. This gives an algorithm with

running-time at most a polynomial multiplied by $3^{\operatorname{size}(T)}$.

\section{BLOCKED SUBMANIFOLDS}

Normal (and almost normal) surfaces cut a triangulated manifold into pieces. These submanifolds have natural polyhedral structures which we now investigate.

Let $\tau$ be a model tetrahedron, and suppose that $S \subset \tau$ is a embedded collection of normal disks and at most one almost normal piece. Let $B$ be the closure of any component of $\tau-S$. We call $B$ a block. See Figure 4.

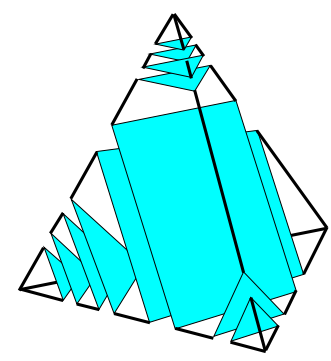

Figure 4. The tetrahedron $\tau$ cut along $S$. Note that in this example there are two blocks of the form "normal disk cross interval". 
A block cobounded by two normally isotopic normal disks is called a product block. All other blocks are called core blocks. Note that there are only seven classes (or types) of product block, corresponding to the seven types of normal disks. Likewise there is a bounded number of core blocks. Five such are shown in Figure 4, but many more are possible. Most of these meet an almost normal annulus.

Suppose that $B$ is a block. The components of $\partial B$ meeting $S$ are the horizontal boundary components of $B$, denoted $\partial_{h} B$. All other faces of $B$ (the faces of $B$ which lie in the two-skeleton) are $\partial_{v} B$, the vertical boundary.

Suppose now that $T$ is a triangulation of a three-manifold and $S \subset|T|$ is a normal or almost normal surface. For simplicity, suppose that $S$ is transversely oriented and separating. Let $N_{S}$ be the closure of the component of $|T|-S$ pointed at by the transverse orientation.

We call $N_{S}$ a blocked submanifold of $|T|$. Let $\widehat{N}_{P}$ be the union of all product blocks in $N_{S}$ and let $\widehat{N}_{C}$ be the union of all core blocks in $N_{S}$.

Remark 5.1. In any blocked submanifold the number of core blocks is at most linear in $\operatorname{size}(T)$. In fact there are at most six in each tetrahedron plus possibly two more coming from the almost normal annulus.

Note that $\widehat{N}_{P}$ and $\widehat{N}_{C}$ need not be submanifolds of $|T|$. To produce submanifolds let $N_{P}$ be a closed regular neighborhood of $\widehat{N}_{P}$, taken inside of $N_{S}$. Also, take $N_{C}$ to be the closure of $N_{S}-N_{P}$. Note the asymmetry between the definitions of $N_{P}$ and $N_{C}$ : we have $\widehat{N}_{P} \subset N_{P}$ while $N_{C} \subset \widehat{N}_{C}$. As above define $\partial_{h} N_{P}=N_{P} \cap S$ and $\partial_{v} N_{P}=$ $\overline{\partial N_{P}-\partial_{h} N_{P}}$. The horizontal and vertical boundaries $\partial_{h} N_{C}$ and $\partial_{v} N_{C}$ are defined similarly.

We now give an efficient encoding of a component $N \subset N_{P}$, similar to the surface vectors $v(S)$. As usual, two blocks $B, B^{\prime}$ of $N$ are of the same type if there is a normal isotopy of the model tetrahedron sending $B$ to $B^{\prime}$. A stack $k$ is all blocks of $N$ of a fixed type. The size of $k$ is the number of blocks it contains. Orienting $k$ gives positions to the blocks in $k$. The block vector $v(N)=\left(v_{k}(N)\right)$ is a vector in $\mathbb{Z}^{7 \cdot \operatorname{size}(T)}$ recording the size of each stack.

We also record the vertical boundary of $N$; note that $\partial_{v} N$, as it is the boundary of a regular neighborhood of $\widehat{N}$, is subdivided into a linear number of vertical rectangles (Remark 5.1). Along with the annuli $\partial_{v} N$ we record the position of every block (or position) in every stack giving rise to a rectangle in $\partial_{v} N$. Taken all together, the block vector, 
the vertical boundary, the positions in the stacks, and the matching equations suffice to recover $N$ up to normal isotopy.

Theorem 5.2. There is a polynomial-time algorithm that, given a triangulation $T$ and a surface vector $v(S)$ for a transversely oriented $S$, produces the block vector $v(N)$, the vertical boundary $\partial_{v} N$, and the stack positions for every connected component $N \subset N_{P} \subset N_{S}$. In addition, for every $N$, every stack $k$, and every position $p$ in $k$ (not necessarily coming from $N$ ) the algorithm computes how p partitions the number $v_{k}(N)$.

Proof. When $S$ is not separating replace $S$ by the horizontal boundary of a regular neighborhood $S \times I \subset|T|$, with transverse orientation pointing outward. Take $N_{S}$ equal to the closure of $|T|-(S \times[0,1])$. In what follows we assume that $S$ is separating.

By Remark 5.1 we may explicitly build the core $N_{C}$ for $N_{S}$. The vertical boundary of $N_{C}$ is the desired collection of annuli.

Every normal disk meets an edge $(0 i)$ of the containing model tetrahedron, minimizing $i$. The transverse orientation on $S$ is equivalent to a bit vector $\left(\epsilon_{k}\right) \in\{0,1\}^{7 \cdot \operatorname{size}(T)}$, as follows. The disk (of type $k$ in $S$ ) closest to the vertex 0 has transverse orientation pointing away from 0 if and only if $\epsilon_{k}=0$. The transverse orientation of the $m^{\text {th }}$ disk of type $k$ now depends only on the parity of $m$. Verifying that $\left(\epsilon_{k}\right)$ gives a consistent transverse orientation on the whole of $S$ is a polynomial number of parity calculations.

Recall that the transverse orientation points into $N_{S}$. Let $v\left(N_{P}\right)$ be the block vector. If $v_{k}(S)$ is odd then $v_{k}\left(N_{P}\right)=\left(v_{k}(S)-1\right) / 2$. If $v_{k}(S)$ is even then $v_{k}\left(N_{P}\right)=\left(v_{k}(S)-2 \epsilon_{k}\right) / 2$. We may now label every rectangle in $\partial_{v} N_{P}$ by its position in the corresponding stack. As in Theorem 4.6 use the extended counting algorithm [2] to split $v\left(N_{P}\right)$ as a sum of block vectors together with vertical boundaries. For any component $N$ of $N_{P}$ the counting algorithm can also detect how any position $p$ in any stack $k$ partitions the number $v_{k}(N)$. We do this by modifying the weight vector to record partitions instead of just weights. See the second paragraph of the proof of Corollary 17 in [2].

Remark 5.3. If $S$ is connected then the number of connected components of $N_{P}$ is at most a linear in $\operatorname{size}(T)$. This is because $\partial_{v} N_{P}=\partial_{v} N_{C}$ and the latter has at most linearly many components. (See Remark 5.1.) This is in pleasant contrast to Theorem 4.6. 


\section{NORMALIZING SLOWLY}

In this section we discuss a restricted version of Haken's normalization procedure for producing normal surfaces. This material appeared first in an unpublished preprint of mine and later in my thesis [25]. I thank Danny Calegari for reading an early version of this work. I also thank Bus Jaco for several enlightening conversations regarding barrier surfaces. For example Lemma 8.1 can also be deduced, via a careful induction argument, from Lemma 3.1 of [16]. In addition to Jaco and Rubinstein [16], other authors have independently produced versions of these ideas such as King [18] and Barchechat [3].

Definition 6.1. Take $S$ a closed orientable surface. Let $C_{0}=S \times[0,1]$. Choose a disjoint collection of simple closed curves in some component of $S \times\{0\}$ and attach two-handles in the usual fashion along these curves. Cap off some (but not necessarily all) of any resulting twosphere boundary components with three-handles. The final result, $C$, is a compression body. Set $\partial_{+} C=S \times\{1\}$ and set $\partial_{-} C=\partial C-\partial_{+} C$.

Our definition differs from others (for example [6]) in that two-sphere components in $\partial_{-} C$ are allowed. The reasons for this are explained in Remark 9.3.

Fix $T$ a triangulation of a closed, orientable, connected three-manifold. Let $S \subset|T|$ be a transversely oriented, almost normal surface.

Definition 6.2. A compression body $C_{S} \subset|T|$ is associated to $S$ if $\partial_{+} C_{S}=S, \partial_{-} C_{S}$ is normal, the transverse orientation points into $C_{S}$, and any normal surface $S^{\prime}$ disjoint from $S$ may be normally isotoped to be disjoint from $C_{S}$.

As a bit of notation take $\operatorname{norm}(S)=\partial_{-} C_{S}$ and call this the normalization of $S$. This is well-defined by the following.

Theorem 10.1. Given a transversely oriented almost normal surface $S$ there exists an associated compression body $C_{S}$ and it is unique up to normal isotopy. Furthermore there is a algorithm that, given the triangulation $T$ and the surface vector $v(S)$, computes the surface vector $v(\operatorname{norm}(S))$.

Remark 6.3. As in Theorem 5.2, when $S$ is not separating we add a parallel copy $S^{\prime}$ and transversely orient away from the parallel region between $S$ and $S^{\prime}$. Henceforth, we will assume that $S$ is in fact separating.

The proof of Theorem 10.1 spans Sections 7 to 10. We here give the necessary definitions. In Section 7 we discuss the tightening procedure. 
In Section 8 we show that the tightening procedure gives an embedded isotopy. We discuss the capping off procedure in Section 9. The proof is finished in Section 10.

6.1. Non-normal surfaces. Let $S$ be a surface properly embedded in a triangulated three-manifold $|T|$ and suppose that $S$ is transverse to the skeleta of $T$. Denote the $i$-skeleton of $T$ by $T^{i}$.

We characterize some of the ways $S$ can fail to be normal. A facial curve of $S$ is a simple closed curve of intersection between $S$ and the interior of some triangular face $f \in T^{2}$. A bent arc of $S$ is a properly embedded arc of intersection between $S$ and the interior of some triangular face $f \in T^{2}$ with both endpoints of the arc contained in a single edge of $f$. Both of these are drawn in Figure 5.

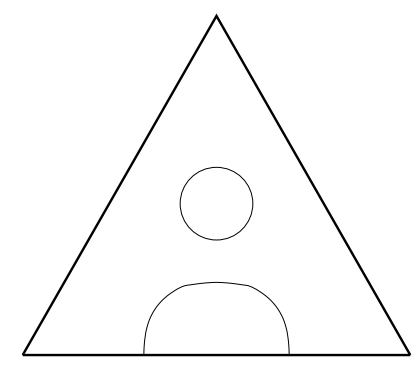

Figure 5. A facial curve and a bent arc.

\subsection{Surgery and tightening disks.}

Definition 6.4. An embedded disk $D \subset|T|$ is a surgery disk for $S$ if

- $D \cap S=\partial D$,

- $D \subset T^{2}$ or $D \cap T^{2}=\emptyset$, and

- $D \cap T^{1}=\emptyset$.

There is a surgery of $S$ along $D$ : Remove a small neighborhood of $\partial D$ from $S$ and cap off the boundaries thus created with disjoint, parallel copies of $D$. Note that we do not require $\partial D$ to be essential in $S$. A facial curve of $S \cap T^{2}$ is innermost if it is the boundary of a surgery disk embedded in a triangle of $T^{2}$.

A bigon is an disk $D$ with given subarcs $\alpha, \beta \subset \partial D$ so that $\alpha \cup \beta=\partial D$ and $\alpha \cap \beta=\partial \alpha=\partial \beta$.

Definition 6.5. An embedded bigon $D \subset|T|$ is a tightening disk for $S$ if

- $D \cap S=\alpha$,

- $D \subset T^{2}$ or $D \cap T^{2}=\beta$, 
- $D \cap T^{1}=\beta$, and

- $D \cap T^{0}=\emptyset$.

There is a tightening isotopy of $S$ across $D$ : Push $\alpha$ along $D$, via ambient isotopy of $S$ supported in a small neighborhood of $D$, until $\alpha$ moves past $\beta$. This procedure reduces weight $(S)$ by exactly two. A bent arc of $S$ is outermost if it lies on the boundary of a tightening disk embedded in a triangle of $T^{2}$.

Suppose $S$ contains an almost normal octagon, $A \subset \tau$. Then there are two tightening disks on opposite sides of $A$ both giving tightening isotopies of $S$ to possibly non-normal surfaces of lesser weight. To see these disks, consult the left-hand side of Figure 3. Notice there are two edges of $\tau$, say $e$ and $e^{\prime}$, so that $|A \cap e|=\left|A \cap e^{\prime}\right|=2$. The first tightening disk has boundary running along $e$ between the points of intersection with $A$; then the boundary runs along an arc in the interior of $A$. The second disk similarly meets $e^{\prime}$. We arrange matters so that the tightening disks lying in the interior of $\tau$ meet each other in a single point.

The above disks are the exceptional tightening disks associated to $A$. If $S$ contains an almost normal annulus then the tube is parallel to at least one edge of the containing tetrahedron. See the right-hand side of Figure 3. For every such edge there is an exceptional tightening disk. Also, the disk which surgers the almost normal annulus will be called the exceptional surgery disk.

\section{Tightening}

This section discusses the tightening procedure which will yield an embedded isotopy. This is proved in Lemma 8.1 below. Fix $T$ is a triangulation of a three-manifold. Suppose that $S \subset|T|$ is a transversely orientable separating almost normal surface. We wish to isotope $S$ off of itself while steadily reducing the weight of $S$.

Suppose that $D$ is an exceptional tightening disk for $S$. Choose the transverse orientation for $S$ which points into the component of $|T|-S$ which meets $D$. The $F$-tightening procedure constructs a map $\mathcal{F}: S \times[0, n] \rightarrow|T|$ as follows:

(1) Let $F_{0}=S$. Take $\mathcal{F}_{0}: S \times\{0\} \rightarrow|T|$ to be projection to the first factor. Let $D_{0}=D$.

(2) Do a small normal isotopy of $F_{0}$ in the transverse direction while tightening $F_{0}$ along $D_{0}$. This extends $\mathcal{F}_{0}$ to a map $\mathcal{F}_{1}: S \times[0,1] \rightarrow|T|$, with $F_{t}=\mathcal{F}_{1}(S \times\{t\})$. Note that the surface $F_{1}$ inherits a transverse orientation from $F_{0}$. Arrange matters so that $F_{\frac{1}{2}}$ is the only level which is not transverse to 
$T^{2}$. Furthermore $F_{\frac{1}{2}}$ only has a single tangency with $T^{1}$ and this tangency occurs in the middle of $\partial D_{0} \cap T^{1}$.

(3) Suppose, at step $k \geq 1$, that $F_{k}$ has an outermost bent arc $\alpha$ with the transverse orientation of $F_{k}$ pointing into the tightening disk $D_{k}$. So $D_{k}$ is cut out of $T^{2}$ by $\alpha$. Extend $\mathcal{F}_{k}$ to $\mathcal{F}_{k+1}: S \times[0, k+$ 1] $\rightarrow|T|$ by doing a small normal isotopy of $F_{k}$ in the transverse direction while tightening $F_{k}$ across $D_{k}$, the $k^{\text {th }}$ tightening disk. So $\mathcal{F}_{k}=\mathcal{F}_{k+1} \mid S \times[0, k]$ and $F_{t}=\mathcal{F}_{k+1}(S \times\{t\})$. Note that the surface $F_{k+1}$ inherits a transverse orientation from $F_{k}$. Arrange matters so that $F_{k+\frac{1}{2}}$ is the $k+1^{\text {th }}$ level which is not transverse to $T^{2}$. Furthermore $F_{k+\frac{1}{2}}$ only has a single tangency with $T^{1}$ and this tangency occurs in the middle of $\partial D_{k} \cap T^{1}$.

(4) Suppose, at step $k \geq 1$, that there is no outermost bent arc $\alpha \subset F_{k}$. Set $n=k$ and halt.

Remark 7.1. As weight $\left(F_{k+1}\right)=$ weight $\left(F_{k}\right)-2$ this tightening procedure terminates after at most weight $(S)$ step. Note also that $\mathcal{F}_{n}$ is far from unique - at any stage in the procedure there may be many tightening disks to choose from.

We will show in Lemma 8.1 that the map $\mathcal{F}_{n}: S \times[0, n] \rightarrow M$ is an embedding. Note that, by construction, $S=F_{0}=\mathcal{F}_{n}(S \times\{0\})$ and in general $F_{t}=\mathcal{F}_{n}(S \times\{t\})$. To simplify notation set $\mathcal{F}=\mathcal{F}_{n}$.

\section{TRACKING ThE ISOTOPY}

Let $S \subset|T|, \mathcal{F}, \mathcal{F}_{k}$, and $F_{t}$ be as defined in Section 7. Suppose that $f$ is any face of any model tetrahedron $\tau \in T$. In this section we analyze how the preimage (in $\tau$ ) of the image of $\mathcal{F}_{k}$ (in $|T|$ ) intersect $f$. We will abuse notation by writing $f \cap \mathcal{F}_{k}$ for this intersection.

Lemma 8.1. For every $k$, the map $\mathcal{F}_{k}$ is an embedding. Furthermore, for $k>0$ and for every face $f \in T^{2}$, the connected components of $f \cap \mathcal{F}_{k}$ are given, up to symmetry, by Figures 6 and 7.

Before proceeding to the proof note that the normal arcs, bent arcs, and facial curves bounding the components shown Figures 6 and 7 inherit a transverse orientation from $S$ or $F_{k}$. In all cases the transverse orientation on $S$ points into the intersection $f \cap \mathcal{F}_{k}$ while the transverse orientation on $F_{k}$ points away. The components of intersection containing a normal arc of $F_{k}$ are called critical. Those with a single bent arc of $F_{k}$ are called temporary. Any component containing a single facial curve of $F_{k}$ is called terminal with a hole. 


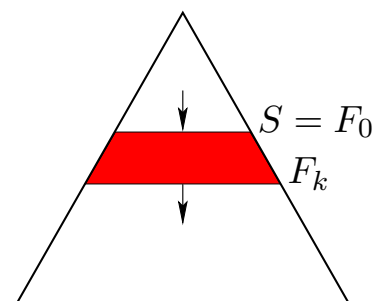

Critical

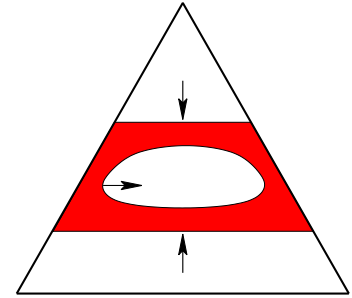

Terminal with a hole

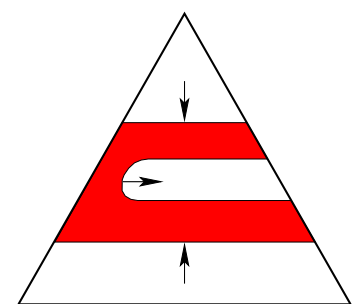

Temporary

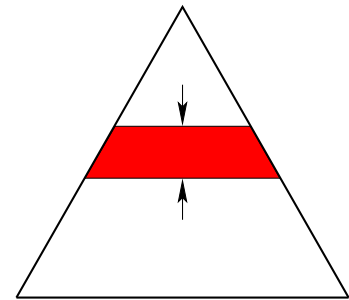

Terminal

Figure 6. The rectangles

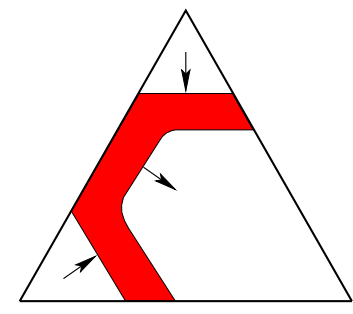

Critical

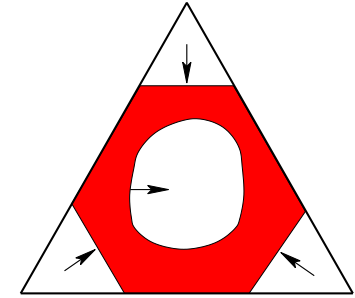

Terminal with a hole

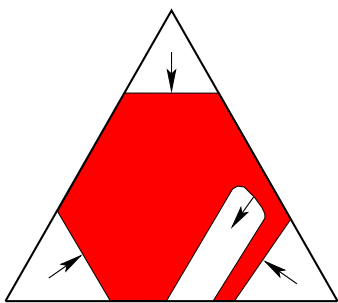

Temporary

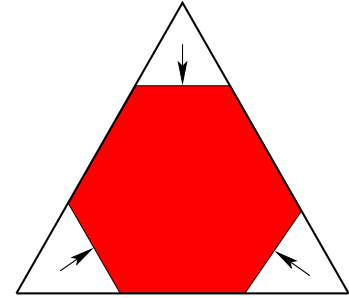

Terminal

FiguRE 7. The hexagons

Finally, components of $f \cap \mathcal{F}_{k}$ which are completely disjoint from $F_{k}$ are simply called terminal. Again, refer to Figures 6 and 7.

The tightening procedure combines the critical components in various ways. However, a temporary component always results in a terminal (possibly with a hole) and these are stable. Note also that there is a 
second critical rectangle with the opposite transverse orientation. The non-critical components may be foliated by the levels of $\mathcal{F}_{k}$ in multiple ways, depending on the order of the tightening isotopies.

Proof of Lemma 8.1. We induct on $k$; both claims are trivial for $k=0$. Now to deal with $k=1$. The exceptional tightening disk $D_{0}$ has interior disjoint from $S=F_{0}$. It follows that $\mathcal{F}_{1}$ is an embedding. To verify the second claim for $k=1$ note that the image of $\mathcal{F}_{1} \mid S \times[0, \epsilon]$ intersects all faces $f \in T^{2}$ only in critical rectangles. Up to $t=\frac{1}{2}$ the image of $\mathcal{F}_{1} \mid S \times[0, t]$ intersected with $f$ is combinatorially constant. Crossing $t=\frac{1}{2}$ adds a regular neighborhood of $D_{0}$ to the image. This only intersects $f$ in a regular neighborhood of $\partial D_{0} \cap T^{1}$. So the pieces of $f \cap \mathcal{F}_{1}$ are unions of critical rectangles connected by small neighborhoods of sub-arcs of $T^{1}$. Also these sub-arcs only meet the $F_{t}$ side of the critical rectangles. As each critical rectangle meets two edges of the face $f$ it follows that at most three critical rectangles are joined together to form a component of $f \cap \mathcal{F}_{1}$. We list all possible cases - consulting Figures 6 and 7 will be helpful:

(1) Two critical rectangles in $f$ combine to produce a temporary rectangle, a terminal rectangle with a hole, or a critical hexagon.

(2) Three critical rectangles in $f$ combine to produce a temporary hexagon or a terminal hexagon with a hole.

Now to deal with the general case: Suppose that both hypotheses hold at stage $k$. Suppose that $\alpha \subset F_{k}$ is the bent arc on the boundary of $D_{k} \subset f \in T^{2}$, the next tightening disk in the sequence. Suppose that interior $\left(D_{k}\right)$ meets image $\left(\mathcal{F}_{k}\right)$. By the second induction hypothesis there is a component, $C$, of $f \cap \mathcal{F}_{k}$ which meets interior $\left(D_{k}\right)$ and appears among those listed in Figures 6 and 7 . Observe that each component of $f \cap \mathcal{F}_{k}$, and hence $C$, meets at least two edges of $f$. The bent arc $\alpha$ meets only one edge of $f$. It follows that the interior of $C$ must meet $\alpha$. Thus $\mathcal{F}_{k}$ was not an embedding, a contradiction.

It follows that $D_{k} \cap$ image $\left(\mathcal{F}_{k}\right)=\alpha$. Since the $k+1^{\text {th }}$ stage of the isotopy is supported in a small neighborhood of $F_{k} \cup D_{k}$ it follows that $\mathcal{F}_{k+1}$ is an embedding.

Now, the transverse orientation on $F_{k}$ gives rise to a transverse orientation on $F_{k+1}$. To verify the second hypothesis we again list the possible cases:

(1) Two critical rectangles in $f$ combine to produce a temporary rectangle, a terminal rectangle with a hole, or a critical hexagon.

(2) Three critical rectangles $f$ combine to produce a temporary hexagon or a terminal hexagon with a hole. 
(3) A critical rectangle and critical hexagon in $f$ combine to produce a temporary hexagon or a terminal hexagon with a hole.

(4) A temporary component leads to a terminal one (possibly with a hole).

This completes the induction.

Remark 8.2. By maximality of $\mathcal{F}$, the surface $F_{n}=\mathcal{F}(S \times\{n\})$ has no outermost bent arcs with outward orientation. A bent arc with inward orientation would violate the second induction hypothesis of Lemma 8.1. So $F_{n}$ contains no bent arcs. $F_{n}$ may contain facial curves, but the second induction hypotheses shows that all of these are innermost with transverse orientation pointing toward the bounded surgery disk.

If we replace $S$ in Lemma 8.1 by a disjoint union of $S$ with a collection of normal surfaces we obtain the following corollary.

Corollary 8.3. If $S^{\prime}$ is any normal surface in $|T|$ which does not intersect $S$ then $\mathcal{F} \cap S^{\prime}=\emptyset$, perhaps after a normal isotopy of $S^{\prime}$ (rel S).

Suppose that $\tau$ is a model tetrahedron in the given triangulation $T$. We again abuse notation, writing $\tau-\mathcal{F}_{k}$ for the complement of the preimage (in $\tau$ ) of the image of $\mathcal{F}_{k}$ (in $|T|$ ).

Lemma 8.4. For all $k \geq 1, \tau-\mathcal{F}_{k}$ is a disjoint collection of balls.

Proof. Our induction hypothesis is as follows: $\tau-\mathcal{F}_{k}$ is a disjoint collection of balls, unless $k=0$ and $\tau$ contains the almost normal annulus of $S$. In that exceptional case $\tau-\mathcal{F}_{0}$ is a disjoint collection of balls and one solid torus.

The base case is trivial. Suppose $B$ is a component of $\tau-\mathcal{F}_{k}$. There are now two cases to consider. Either $B$ is cut by an exceptional tightening disk or it is not. Assume the latter. Then $B$ is a three-ball by induction and after the $k+1^{\text {th }}$ stage of the isotopy $B \cap \mathcal{F}_{k+1}$ is a regular neighborhood (in $B$ ) of a collection of disjoint arcs and disks in $\partial B$. Hence $B-\mathcal{F}_{k+1}$ is still a ball.

If $B$ is adjacent to the almost normal piece of $F_{0}$ then let $D_{0}$ be the exceptional tightening disk. Set $B_{\epsilon}=B-\operatorname{neigh}\left(D_{0}\right)$. Each component of $B_{\epsilon}$ is a ball, and the argument of the above paragraph shows that they persist in the complement of $\mathcal{F}_{1}$.

Recall that $\partial \mathcal{F}_{k}=S \cup F_{k}$. As usual, for a model tetrahedron $\tau$ we write $\tau \cap F_{k}$ for the preimage of $F_{k}$ in $\tau$. A corollary of Lemma 8.4 is the following. 
Corollary 8.5. For all $k$, the connected components of $\tau \cap F_{k}$ are planar.

The connected components of $\tau \cap F_{n}$ warrant closer attention.

Lemma 8.6. If $n \geq 1$ then each component of $\tau \cap F_{n}$ has at most one normal curve boundary component. This normal curve must be short.

Proof. Let $\tau \in T$ be a tetrahedron. Let $P$ be a connected component of $\tau \cap F_{n}$. By Lemma 8.1 the boundary $\partial P$ is a collection of facial curves and normal curves in $\partial \tau$. Let $\alpha$ be any normal curve in $\partial P$. Let $\left\{\alpha_{j}\right\}$ be the normal arcs of $\alpha$.

Claim. $\alpha$ has length three or four.

Proof of Claim. Call the collection of critical rectangles and hexagons in $\partial \tau \cap \mathcal{F}$ that meet $\alpha$ the support of $\alpha$. To prove the claim we have two cases. First suppose that only critical rectangles support $\alpha$. So $\alpha$ is normally isotopic to a normal curve $\beta \subset \partial \tau \cap S$. The first step of the tightening procedure prevents $\beta$ from being a boundary of the almost normal piece of $S$. It follows that $\alpha$ must be short.

Otherwise $\alpha_{1}$, a normal subarc of $\alpha$, is on the boundary of a critical hexagon $h \subset f$. Let $\beta$ be a normal curve of $S$ meeting $h$ and let $\beta_{1} \subset \beta$ be one of the normal arcs in $\partial h$. Let $e$ be the edge of $f$ which $\alpha_{1}$ does not meet. This edge is partitioned into three pieces; $e_{h} \subset h, e^{\prime}$, and $e^{\prime \prime}$. We may assume that $\beta_{1}$ separates $e_{h}$ from $e^{\prime}$. See Figure 8.

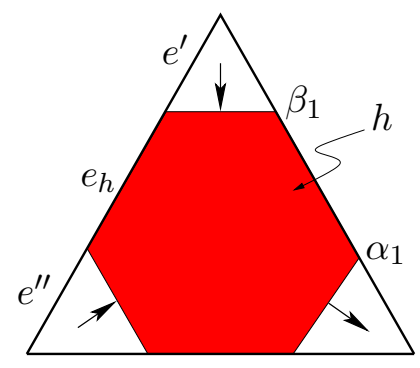

FiguRE 8 . The normal arcs $\alpha_{1}$ and $\beta_{1}$ are on the boundary of the critical hexagon $h$. Note that $\beta$ does not meet $e^{\prime}$ or interior $(h)$.

Note that a normal curve of length $\leq 8$ has no parallel normal arcs in a single face. Thus $\beta$ meets $e^{\prime}$ exactly once at an endpoint of $e^{\prime}$. Since $\alpha$ and $\beta$ do not cross it follows that $\beta$ separates $\alpha$ from $e^{\prime}$ in $\partial \tau$.

Similarly, $\alpha$ is separated from $e^{\prime \prime}$. Thus $\alpha$ does not meet $e$ at all. By Lemma 4.2 the normal curve $\alpha$ is short. This finishes the proof of the claim. 
Claim. The component $P \subset \tau \cap F_{n}$ has at most one boundary component which is a normal curve.

Proving this will complete the lemma. So suppose that $\partial P$ contains two normal curves: $\alpha$ and $\beta$. Let $A$ be the annulus cobounded by $\alpha$ and $\beta$ in $\partial \tau$, the boundary of the model tetrahedron.

Suppose now that the transverse orientation, that $\alpha$ inherits from $F_{n}$, points away from $A$. Thus $A$ and the support of $\alpha$ intersect. There are several cases to examine, depending on the length of $\alpha$ and the components of the support of $\alpha$.

(1) Suppose $\alpha$ has length three:

(a) If only critical rectangles support $\alpha$ then a normal triangle of $S$ separates $\alpha$ and $\beta$.

(b) If one critical hexagon and two critical rectangles support $\alpha$ then the almost normal octagon and the exceptional tightening disk together separate $\alpha$ and $\beta$. See left hand side of Figure 9.

(c) If two critical hexagons and one critical rectangle support $\alpha$ then a normal triangle or a normal quad of $S$ separates $\alpha$ from $\beta$. See right hand side of Figure 9 .

(d) If only critical hexagons support $\alpha$ then a normal triangle of $S$ separates $\alpha$ and $\beta$.

(2) Suppose $\alpha$ has length four:

(a) If only critical rectangles support $\alpha$ then a normal quad of $S$ separates $\alpha$ and $\beta$.

(b) If one critical hexagon and three critical rectangles support $\alpha$ then $S$ could not have been an almost normal surface. See left hand side of Figure 10.

(c) If two critical hexagons and two critical rectangles support $\alpha$ then a normal triangle of $S$ separates $\alpha$ and $\beta$. See right hand side of Figure 10.

When $\alpha$ has length four it cannot be supported by more than two critical hexagons.

To recap: in all cases except 1(b) and 2(b), the support of $\alpha$ (possibly together with a terminal rectangle or hexagon) closes up, implying the existence of a normal disk of $S$ with boundary a core curve of the annulus $A$. As this disk lies in $S$ observe that $S \cap P \neq \emptyset$ and thus $S \cap F_{n} \neq \emptyset$. This contradicts the fact that $\mathcal{F}$ is an embedding (Lemma 8.1). Case 1(b) is similar, except that the support of $\alpha$ meets other critical or terminal components to form the octagon piece of $S$. So $P$ must intersect either $S$ or the exceptional tightening disk, again 


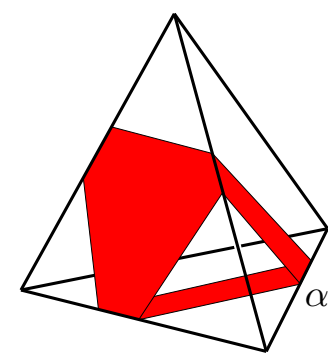

One hexagon

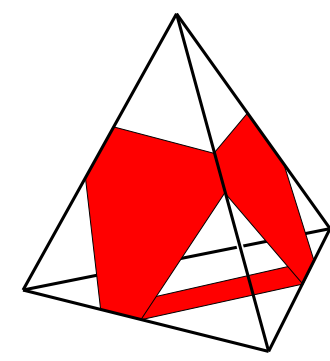

Two hexagons

Figure 9. Diagrams for cases (1b) and (1c).

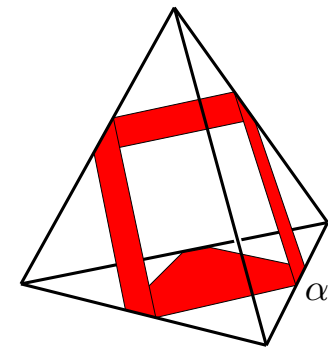

One hexagon

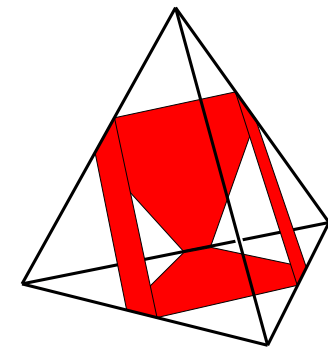

Two hexagons

Figure 10. Diagrams for cases (2b) and (2c).

a contradiction of Lemma 8.1. Lastly, in case 2(b), $S$ could not have been almost normal.

So deduce that the transverse orientation that $\alpha$ inherits from $F_{n}$ must point toward $A$. Thus $A$ and the support of $\alpha$ are disjoint. Let $\gamma$ be an arc which runs along $P$ from $\alpha$ to $\beta$. Let $\alpha^{\prime}$ be a push-off of $\alpha$ along $A$, towards $\beta$. This push-off bounds a disk in one of the components of $\tau-\mathcal{F}$, by Lemma 8.4. This disk does not intersect $P \subset F_{n} \subset \mathcal{F}$ and hence fails to intersect $\gamma$. This is a contradiction.

Remark 8.7. By Lemma 8.1 all facial curves of $F_{i}$ are innermost. It follows that the "tubes" analyzed in Lemma 8.6 do not run through each other.

\section{CApping ofF}

Here we construct our candidate for $C_{S}$, the compression body associated to $S$.

Let $\mathcal{F} \subset|T|$ be the image of the map constructed above. Recall that $\partial \mathcal{F}=S \cup F_{n}$ where $S$ is the almost normal surface we started with and 
$F_{n}$ is the surface obtained by "tightening" $S$. Note that, since $\mathcal{F}$ is the embedded image of $S \times[0, n]$, in fact $F_{n}$ is isotopic to $S$ in $|T|$. They are not normally isotopic as weight $\left(F_{n}\right)<\operatorname{weight}(S)$.

Definition 9.1. A two-sphere which is embedded in $|T|$ but disjoint from $T^{2}$ is called a bubble.

From Lemma 8.6, Corollary 8.5, and Remark 8.7 we deduce:

Corollary 9.2. Let $F_{n}^{\prime}$ be the surface obtained by surgering all facial curves of $F_{n}$. Then $F_{n}^{\prime}$ is a disjoint collection of bubbles and normal surfaces. Each bubble bounds a ball with interior disjoint from $T^{2} \cap$ $F_{n}^{\prime}$.

Construct $C_{S}$ as follows: For every facial curve $\alpha$ of $F_{n}$ attach a two-handle to $\mathcal{F}$ along $\alpha$. Attach so that the core of the two-handle is the subdisk of $T^{2}$ cut out by $\alpha$. Call this $\mathcal{F}^{\prime}$. As noted in Remark 8.7 all facial curves of $F_{n}$ are innermost. So $\mathcal{F}^{\prime}$ is an embedded compression body. At this point there may be components of $\partial_{-} \mathcal{F}^{\prime}$ which are not normal. By Corollary 9.2 all of these are bubbles bounding a ball disjoint from all of the other bubbles. Cap off each bubble to obtain $C_{S}$. Set $\operatorname{norm}(S)=\partial_{-} C_{S}$. The next section proves that $v(\operatorname{norm}(S))$ does not depend on the choices made in the construction of $\mathcal{F}$.

Remark 9.3. Normal two-spheres may appear in the normalization procedure. In particular, if $S$ is an almost normal two-sphere then, for one of the two possible transverse orientations, there will always be a normal two-sphere appearing in $\operatorname{norm}(S)$. This is why two-spheres are permitted in $\partial_{-} C$ in Definition 6.1.

\section{Proof of The Normalization THEOREM}

Suppose that $S$ is almost normal and equipped with a transverse orientation. Recall from Definition 6.2 that $C_{S}$, a compression body in $|T|$, is associated to $S$ if $\partial_{+} C_{S}=S, \partial_{-} C_{S}$ is normal, the transverse orientation on $S$ points into $C_{S}$, and any normal surface $S^{\prime} \subset|T|$ disjoint from $S$ may be normally isotoped to one disjoint from $C_{S}$.

We now have the following.

Theorem 10.1. Given a transversely oriented almost normal surface $S$ there exists a compression body $C_{S}$ associated to $S$ and $C_{S}$ is unique (up to normal isotopy). Furthermore there is a algorithm that, given the triangulation $T$ and the surface vector $v(S)$, computes the surface vector of $\operatorname{norm}(S)=\partial_{-} C_{S}$.

Proof. We proceed in several steps. 
Claim. There exists a compression body $C_{S}$ associated to $S$.

Proof. There are two cases. Either the transverse orientation for $S$ points at the exceptional surgery disk (implying that $S$ contained an almost normal annulus) or the transverse orientation points at an exceptional tightening disk.

In the first case, $C_{S}$ is obtained by thickening $S$ slightly and adding a regular neighborhood of the exceptional surgery disk. It is clear that $C_{S}$ is a compression body, $\partial_{+} C_{S}=S$, and $\partial_{-} C_{S}$ is normal. Suppose that $S^{\prime}$ is any normal surface in $T$ which is disjoint from $S$. Then, perhaps after a normal isotopy of $S^{\prime}(\operatorname{rel} S)$, we have that $S^{\prime}$ is disjoint from the exceptional surgery disk for $S$. It follows that $S^{\prime}$ may be isotoped out of $C_{S}$.

In the second case the transverse orientation of $S$ points at an exceptional tightening disk of $S$. As in Section 7 form $\mathcal{F}$ with $\partial \mathcal{F}=S \cup F_{n}$. As in Section 9 attach two-handles to $\mathcal{F}$ along the facial curves of $F_{n}$ to obtain $\mathcal{F}^{\prime}$. Cap off the bubbles with their three-balls to obtain $C_{S}$. Again, $C_{S}$ is a compression body with $\partial_{+} C_{S}=S$.

Suppose now that $S^{\prime}$ is some normal surface in $T$ which is disjoint from $S$. Then, by Corollary 8.3, the surface $S^{\prime}$ is disjoint from $\mathcal{F}$, perhaps after a normal isotopy of $S^{\prime}$ (rel $S$ ). Since $S^{\prime}$ is normal it cannot meet any of the disks (in $T^{2}$ ) bounded by facial curves of $F_{n}$. So $S^{\prime} \cap \mathcal{F}^{\prime}=\emptyset$ as well. Finally, suppose that $A$ is a bubble component of $\partial_{-} \mathcal{F}^{\prime}$. Let $B$ be the three-ball which $A$ bounds (such that $B \cap T^{2}=\emptyset$ ). Then no component of $S^{\prime}$ meets $B$ as $S^{\prime} \cap A=\emptyset$ and $S^{\prime}$ is normal. Deduce that $S^{\prime} \cap C_{S}=\emptyset$. This finishes the claim.

Claim. The associated compression body $C_{S}$ is unique (up to normal isotopy).

Proof. Suppose that $C_{S}$ and $C_{S}^{\prime}$ are both associated to $S$. Let $A=\partial_{-} C_{S}$ and $A^{\prime}=\partial_{-} C_{S}^{\prime}$. Then $A$ and $A^{\prime}$ are normal surfaces, both disjoint from $S$. It follows that there exists a normal isotopy $\mathcal{H}$ which moves $A^{\prime}$ out of $C_{S}($ rel $S)$ and another normal isotopy $\mathcal{H}^{\prime}$ which moves $A$ out of $C_{S}^{\prime}$ $(\operatorname{rel} S)$.

Consider any face $f \in T^{2}$ and any normal arc $\alpha \subset f \cap S$. Let $X \subset f \cap C_{S}$ be the component containing $\alpha$. Take $X^{\prime}$ to be the component of $f \cap C_{S}^{\prime}$ which contains $\alpha$. We must show that $X$ and $X^{\prime}$ have the same combinatorial type. Suppose not. After possibly interchanging $X$ and $X^{\prime}$ there are only six situations to consider:

(1) $X$ is a critical rectangle and $X^{\prime}$ is a terminal rectangle.

(2) $X$ is a critical rectangle and $X^{\prime}$ is a critical hexagon.

(3) $X$ is a critical rectangle and $X^{\prime}$ is a terminal hexagon. 
(4) $X$ is a critical hexagon and $X^{\prime}$ is a terminal hexagon.

In any of these four cases let $\delta$ be the normal arc of $A=\partial_{-} C_{S}$ on the boundary of $X$. Note that $\partial X^{\prime}$ contains $\alpha$ (as does $\partial X$ ) and also another normal arc $\beta \subset f \cap S$ which does not meet $X$ (as $S=\partial_{+} C_{S}$ ). Now note that it is impossible for $\mathcal{H}^{\prime}$ to normally isotope $\delta$ out of $X^{\prime}$ while keeping $S$ fixed pointwise (as $\delta$ would have to cross $\beta$ ).

(5) $X$ is a terminal rectangle and $X^{\prime}$ is a critical hexagon.

(6) $X$ is a terminal rectangle and $X^{\prime}$ is a terminal hexagon.

In either of these cases let $\beta$ be the other normal arc of $S \cap \partial X$. Then $\beta$ intersects the interior of $X^{\prime}$, a contradiction. This proves the claim.

Claim. There is a algorithm that, given the triangulation $T$ and the surface vector $v(S)$, computes the surface vector of $\partial_{-} C_{S}=\operatorname{norm}(S)$.

Proof. We follow the proof of Lemma 8.1: We keep track of the intersection $f \cap \mathcal{F}_{k}$ for every face $f$ of every model tetrahedron $\tau$. The intersection is a union of components, with all allowable types shown (up to symmetry) in Figures 6 and 7 . There is at most one hexagon in each face and perhaps many rectangles, arranged in three families, one for each vertex of $f$. At stage $n$ there are no bent arcs remaining. Now delete all facial curves of $F_{n}$ and all normal arcs of $S$. The normal arcs left completely determine $\operatorname{norm}(S)$ and from this we may find the surface vector $v(\operatorname{norm}(S))$. This proves the claim.

Thus we are done with the proof of Theorem 10.1.

The algorithm just given is inefficient. It depends polynomially on $\operatorname{size}(T)$ and weight $(S)$. In the next section we improve this to a algorithm which depends polynomially on $\operatorname{size}(T)$ and $\log (\operatorname{weight}(S))$.

As a corollary of Theorem 10.1:

Corollary 10.2. If $S \subset|T|$ is a transversely oriented almost normal two-sphere then $C_{S}$ is a three-ball, possibly with some open three-balls removed from its interior. These have closures disjoint from each other and from $S$.

Now an orientable surface in an orientable three-manifold may be transversely oriented in exactly two ways. By Theorem 10.1, if $S$ is an almost normal surface, for each transverse orientation there is a associated compression body. Call these $C_{S}^{+}$and $C_{S}^{-}$.

From Corollary 10.2 deduce:

Theorem 10.3. If $S \subset|T|$ is an almost normal two-sphere and both $\partial C_{S}^{+}-S$ and $\partial C_{S}^{-}-S$ are (possibly empty) collections of vertex-linking two-spheres, then $|T|$ is the three-sphere. 
Proof. By hypothesis $\partial C_{S}^{+}-S$ is a collection of vertex linking spheres. For each of these add to $C_{S}^{+}$the corresponding vertex neighborhood. Let $\mathbb{B}^{+}$be the resulting submanifold of $|T|$. By the Alexander trick $\mathbb{B}^{+}$ is a three-ball. Do the same to $C_{S}^{-}$to produce $\mathbb{B}^{-}$. The Alexander trick now implies that the manifold $|T|=\mathbb{B}^{+} \cup_{S} \mathbb{B}^{-}$is homeomorphic to the three-sphere.

\section{An EXAMPLE}

Here we give a brief example of the normalization procedure. Let $T$ be the one vertex triangulation shown in Figure 11.

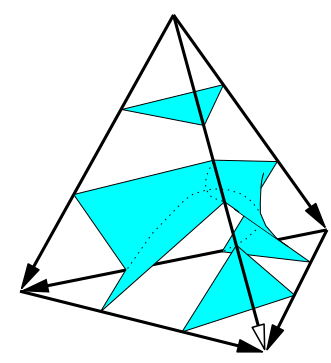

Figure 11. A one-tetrahedron triangulation of $S^{3}$. It is straight-forward to list all normal and almost normal surfaces in $T$. It is a pleasant exercise to draw the graph $T^{1}$ as it actually sits in $S^{3}$. It is somewhat harder to draw the two-skeleton.

The front two faces (1 and 2) are glued to each other as are the back faces $(0$ and 3$)$. The faces are glued to give the edge identifications shown. The surface $S$ depicted in $T$ is an almost normal two-sphere with two triangles and one almost normal octagon.

The sphere $S$ has two exceptional tightening disks: $D$ meeting the edge $(0,3)$ of the model tetrahedron and $D^{\prime}$ meeting edge $(1,2)$.

Tightening along $D$ gives $F_{1}$ which is the vertex link. Tightening along $D^{\prime}$ and then along bent arcs to obtain an $F$-tightening sequence $F_{1}^{\prime}, F_{2}^{\prime}, F_{3}^{\prime}$. As a note of caution: $F_{1}^{\prime}$ drawn in the model tetrahedron has four bent arcs - however $F_{1}^{\prime} \cap T^{2}$ contains only two. These are independent of each other and doing these moves in some order gives $F_{2}^{\prime}$ and $F_{3}^{\prime}$. Then $F_{3}^{\prime}$ is a weightless two-sphere in $T$ with a single facial curve and no other intersection with the two-skeleton. Finally, surger the facial curve of $F_{3}^{\prime}$ and cap off the two resulting bubbles. 
Thus: on the $D$ side of $S$ the normalization is the vertex link. On the $D^{\prime}$ side the normalization is the empty set. It follows from Theorem 10.3 that $|T|$ is the three-sphere.

\section{Normalizing QUICKLY}

The normalization procedure can be accelerated. Suppose that $T$ is a triangulation of a three-manifold and $S$ is assumed to be a transversely oriented almost normal surface.

Theorem 12.1. There is a polynomial-time algorithm that, given any such $T$ and surface vector $v(S)$, produces as output $v(\operatorname{norm}(S))$, the normalization of $S$.

As in Remark 6.3 we will restrict to the case where $S$ is separating. Recall that $N_{S}$ is the closure of the component of $|T|-S$ into which the transverse orientation points. Then $\widehat{N}_{P}$ is the union of all product blocks in $N_{S}$ and $\widehat{N}_{C}$ is the union of all the core blocks. Also $N_{P}$ is a regular neighborhood of $\widehat{N}_{P}$, taken in $N_{S}$. Finally $N_{C}=\overline{N_{S}-N_{P}}$. We will prove Theorem 12.1 by altering our original normalization procedure three times. First we will show that the order of the tightening moves is irrelevant. Then we will show that surgeries on facial curves and capping off of bubbles may happen during the normalization procedure, instead of being held until the end. Finally we show that tightening inside of $N_{P}$ can be done quickly. These three modifications combine to give an efficient algorithm.

12.1. Changing the order of the tightening moves. As stated in Remark 7.1 the isotopy $\mathcal{F}: S \times[0, n] \rightarrow M$ need not be unique. However the first sentence of Theorem 10.1 implies the following.

Lemma 12.2. Any order for the tightening moves (performed in the construction of $\mathcal{F}$ ) gives the same surface $\operatorname{norm}(S)$ once the facial curves of $F_{n}$ have been surgered and bubbles have been capped off.

12.2. Surgery on facial curves and deleting bubbles. We now alter the tightening procedure in a more substantial fashion.

Recall that $S \subset|T|$ is a transversely orientable separating almost normal surface. Recall that $D$ is the exceptional tightening disk for $S$. Transversely orient $S$ to point into the component of $|T|-S$ which meets $D$. Here is the $G$-tightening procedure:

(1) Let $G_{0}=S$. Let $D_{0}=D$.

(2) Do a small normal isotopy of $G_{0}$ in the transverse direction while tightening $G_{0}$ along $D_{0}$. Call the surface so obtained $G_{0}^{\prime}$. Surger all facial curves of $f \cap G_{0}^{\prime}$ for every $f \subset T^{2}$ to obtain $G_{0}^{\prime \prime}$. Delete 
any bubble components of $G_{0}^{\prime \prime}$ (two-sphere components which are contained in the interior of tetrahedra). Call the resulting surface $G_{1}$. Note that $G_{1}$ inherits a transverse orientation from $G_{0}$.

(3) Suppose, at step $k \geq 1$, that $G_{k}$ has an outermost bent arc $\alpha$ with the transverse orientation of $G_{k}$ pointing into the tightening disk $D_{k}$. So $D_{k}$ is cut out of $T^{2}$ by $\alpha$. Perform a small normal isotopy of $G_{k}$ in the transverse direction while tightening $G_{k}$ across $D_{k}$. Call the surface so obtained $G_{k}^{\prime}$. Surger all facial curves of $f \cap G_{k}^{\prime}$ for every $f \in T^{2}$ to obtain $G_{k}^{\prime \prime}$. Delete any bubble components of $G_{k}^{\prime \prime}$. Call the resulting surface $G_{k+1}$. Note that $G_{k+1}$ inherits a transverse orientation from $G_{k}$.

(4) Suppose, at step $k \geq 1$, that there is no outermost bent arc $\alpha \subset G_{k}$. Set $n=k$ and halt.

Lemma 12.3. The surface $G_{n}$ is normally isotopic to $\operatorname{norm}(S)$, the normalization of $S$.

Proof. Recall that Lemma 8.1 gives a complete classification of the possible components of intersection of image $\left(\mathcal{F}_{k}\right)$ with the faces of $T^{2}$. Again, see Figures 6 and 7 . The only components containing a facial curve are the terminal rectangle with hole and terminal hexagon with a hole.

Since the terminal rectangles and hexagons with a hole do not contain normal or bent arcs of $F_{k}$ they remain unchanged in the $F$-tightening procedure until $F_{n}$ is reached. Then all facial curves are surgered and bubbles capped off. Thus it makes no difference to the resulting surface $\operatorname{norm}(S)$ if we surger facial curves and delete bubbles as soon as they appear.

12.3. Tightening in $I$-bundle regions. We now give the final modification of the tightening procedure. Suppose that $v(S)$ is an almost normal surface vector. Suppose also that $S$ has a transverse orientation pointing at an exceptional tightening disk.

Recall that $N_{S}$ is the blocked submanifold cut from $|T|$ by the surface $S$ (so that the transverse orientation points into $N_{S}$ ). Also, $N_{P}$ is the $I$-bundle region of $N_{S}$ while $N_{C}=\overline{N_{S}-N_{P}}$ is the core of $N_{S}$.

We now introduce the final data structures required in the proof$\operatorname{core}(S)$, annuli $(S)$, and product $(S)$ - closely following Section 5 and Theorem 5.2.

Put a copy of the horizontal boundary of $N_{C}$ in core $(S)$. That is, record in $\operatorname{core}(S)$ all of the gluing information between edges of disks which are in the horizontal boundary of core blocks. 
Next, place copies of all components of $\partial_{v} N_{C}$ into annuli $(S)$ and record how they meet the surfaces contained in $\operatorname{core}(S)$.

Finally, define product $(S)$ to be the list $\{2 \cdot v(N)\}$. Here $N$ ranges over the components of $N_{P}$ and $v(N)$ is the corresponding block vector found by Theorem 5.2. We record, using positions in stacks, how the components of $\partial_{v} N_{P}$ are identified with the components of annuli $(S)$. We also record, for each component $N \subset N_{P}$, how each position partitions the numbers $v_{k}(N)$.

We now turn to constructing a sequence of surfaces $H_{k}$. Each $H_{k}$ will be represented by core $\left(H_{k}\right)$, annuli $\left(H_{k}\right)$, and $\operatorname{product}\left(H_{k}\right)$. Here is the $H$-tightening procedure.

(1) Let $\operatorname{core}\left(H_{0}\right)=\operatorname{core}(S)$, annuli $\left(H_{0}\right)=\operatorname{annuli}(S)$, and $\operatorname{product}\left(H_{0}\right)=$ $\operatorname{product}(S)$. Let $D_{0}=D$. Suppose now that we are at step $k$ of the procedure and there is a tightening disk $D_{k}$ for $H_{k}$.

(2) If $D_{k}$ is disjoint from annuli $\left(H_{k}\right)$, and so does not meet $N_{P}$, then perform the tightening move as in the $G$-sequence. This effects only the pieces in $\operatorname{core}\left(H_{k}\right)$ and we use the tightening move to compute core $\left(H_{k+1}\right)$. Set annuli $\left(H_{k+1}\right)=\operatorname{annuli}\left(H_{k}\right)$, $\operatorname{product}\left(H_{k+1}\right)=\operatorname{product}\left(H_{k}\right)$ and go to stage $k+1$.

(3) Suppose $D_{k}$ meets some component of annuli $\left(H_{k}\right)$. Thus $D_{k}$ meets a component of $N_{P}$; call this component $N$. Obtain $\operatorname{product}\left(H_{k+1}\right)$ by deleting the vector $2 \cdot v(N)$ from $\operatorname{product}\left(H_{K}\right)$. To get annuli $\left(H_{k+1}\right)$ delete $\partial_{v} N$ from $\operatorname{annuli}\left(H_{k}\right)$. For each stack $\ell$ and positions $p$ contained in $\ell$ : update the position of $p$ using the partition of $v_{\ell}(N)$ determined by $p$.

Set $\operatorname{core}^{\prime}\left(H_{k}\right)=\operatorname{core}\left(H_{k}\right) \cup \partial_{v} N$. Let $D_{k}^{\prime}=D_{k}-N$; that is, remove a small neighborhood of $T^{1}$ from $D_{k}$. See Figure 12 . Then $D_{k}^{\prime}$ is a surgery disk for $\operatorname{core}^{\prime}\left(H_{k}\right)$. So surger along $D_{k}^{\prime}$, surger along all facial curves of $\operatorname{core}^{\prime}\left(H_{k}\right)$, and delete all bubbles in $\operatorname{core}^{\prime}\left(H_{k}\right)$. This finally yields $\operatorname{core}\left(H_{k+1}\right)$. Go on to stage $k+1$.

(4) If at stage $k$ there is no tightening disk then set $n=k$. Sum the vectors in product $\left(H_{n}\right)$ and add to this vector the number of normal disks of each type in $\operatorname{core}\left(H_{n}\right)$. Output the final sum $v\left(H_{n}\right)$.

This completes the description of the $H$-tightening procedure.

\subsection{Correctness and efficiency.}

Proof of Theorem 12.1. Note that if the transverse orientation on $S$ points towards an exceptional surgery disk of $S$ then the theorem is trivial. So suppose instead that a tightening disk is pointed at. 


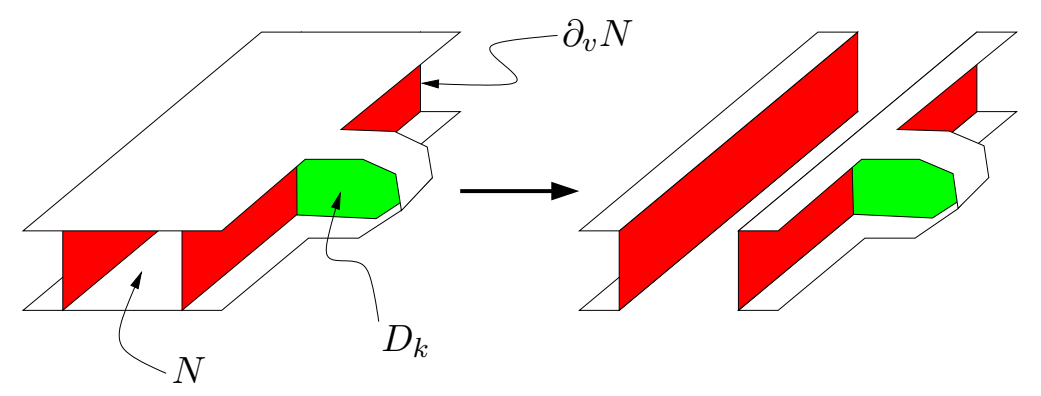

Figure 12. Removing the horizontal boundary of $N$ and adding the vertical.

Claim. The $H$-tightening procedure outputs $v(\operatorname{norm}(S))$.

Proof. It suffices to show that $H_{n}$ is normally isotopic to $\operatorname{norm}(S)$. Suppose that we are given $k, \ell$ so that $G_{k}=H_{\ell}$, perhaps after a normal isotopy. Let $D$ be the given tightening disk. If $D$ does not meet the product region then $G_{k+1}=H_{\ell+1}$ and we are done.

Suppose instead that $D$ meets the product region. Recall that $\partial D=$ $\alpha \cup \beta$ where $\beta \subset T^{1}$. The arc $\beta$ is contained in $T^{1} \cap \partial_{v} \widehat{N}_{P}$ while only a small neighborhood of $\partial \alpha$ (taken in $\alpha$ ) is contained in $N_{P}$. Suppose that $N$ is the component of $N_{P}$ containing $\beta$.

We now show that we can reorder the tightening moves in the $G$ procedure so that there is a $k^{\prime}$ with $G_{k+k^{\prime}}=H_{\ell+1}$. It then follows from Lemma 12.3 that the $H$ procedure produces $v(\operatorname{norm}(S))$.

Recall that $\widehat{N}$ and $N$ are $I$-bundles. Let $\pi$ be the bundle map crushing fibers to a point. Let $E=\pi(N)$. Let $\widehat{E}=\pi(\widehat{N})$. Note that $\widehat{E}$ is not necessarily a surface. However $E$ is a surface with boundary, $\widehat{E}$ naturally embeds in $E$, and there is a small deformation retraction of $E$ to $\widehat{E}$. Note that $\widehat{E}$ and $E$ inherit cell structures from $\widehat{N}$ and $N$. Choose a spanning tree $U$ for the one-skeleton $\widehat{E}^{1}$ of $\widehat{E}$ rooted at $b=\pi(\beta)$. Choose an ordering of the vertices of $U, \sigma: U^{0} \rightarrow\left(\mathbb{N} \cap\left[1, k^{\prime}\right]\right)$, so that for any vertex $d$ with parent $c$ we have $\sigma(c)<\sigma(d)$. Here $k^{\prime}=\left|U^{0}\right|$ is the number of vertices in $U^{0}$.

We now have a sequence of tightening moves to perform in the $G$ procedure. At step one do the tightening move along the disk $D$, surger all facial curves, and delete bubbles. At step $i>1$, examine the edge $e$ between $c$ and $d$ (where $\sigma(d)=i$ and $c$ is the parent of $d$ ). Then, by induction and the fact that $\sigma(c)<\sigma(d)=i$ there is a bent arc of $G_{k+i-1}$ in the rectangle $\pi^{-1}(e)$ with endpoints on the segment $\pi^{-1}(d) \subset T^{1}$. Do this tightening move, surger facial curves, delete bubbles, and go to step $i+1$. 
After $k^{\prime}=\left|U^{0}\right|$ steps we obtain the surface $G_{k+k^{\prime}}$ which is normally isotopic to the following: $\left(G_{k}-\partial_{h} N\right) \cup \partial_{v} N$ surgered along the disk $D^{\prime}$, surgered along facial curves, with bubbles deleted. Here $D^{\prime}=\overline{D-N}$. So $G_{k+k^{\prime}}$ agrees with $H_{\ell+1}$ and the claim is proved.

Claim. Precomputation for the $H$ procedure takes time at most polynomial in $\operatorname{size}(T)$ and $\log (\operatorname{weight}(S))$.

Proof. This follows from Remark 5.1 and Theorem 5.2.

Claim. The number of steps of the $H$ procedure is at most linear in $\operatorname{size}(T)$.

Proof. Each step reduces the weight of core $\left(H_{k}\right)$ by two or removes a vector from product $\left(H_{k}\right)$. Since the weight of $\operatorname{core}\left(H_{k}\right)$ is at most linear (Remark 5.1), and since there are at most a linear number of components of $N_{P}$ (Remark 5.3), the claim follows.

Claim. Performing each step of the $H$ procedure takes time at most polynomial in $\operatorname{size}(T)$ and $\log (\operatorname{weight}(S))$.

Proof. If there is a tightening disk $D_{k}$ contained in $\operatorname{core}\left(H_{k}\right)$ then it can be found in polynomial time.

If $D_{k}$ is disjoint from $N_{P}$ then we only have to alter core $\left(H_{k}\right)$ in the tetrahedra adjacent to $D_{k}$. There are at most a linear number of such tetrahedra.

If the tightening disk meets a component $N \subset N_{P}$ then updating the positions in all stacks is at most a polynomial amount of arithmetic. As in the disjoint case, $\operatorname{core}^{\prime}\left(H_{k}\right)$ is at most linear in size. It follows that surgering facial curves and deleting bubbles can be done in polynomial time.

Thus we can compute the desired result, $v(\operatorname{norm}(S))$, in time which is at most a product of polynomials in $\operatorname{size}(T)$ and $\log ($ weight $(S))$. This completes the proof of Theorem 12.1 .

\section{Crushing: "NeW triangulations FOR OlD"}

Crushing triangulations along normal surfaces is an important step in Casson's algorithm [5]. As usual, we refer the reader to Jaco and Rubinstein's work [16]. The notion of crushing is also explained in detail in Chapter 3 of Barchechat's thesis [3].

Let $T$ be a triangulation of a closed three-manifold. Let $\tau_{i}$ be a model tetrahedron. Fix attention on the quad in $\tau$ partitioning the vertices $\{0,1,2,3\}$ into $\{0, a\}$ and $\{b, c\}$. We say that the quad is of type $a$. 
Let $\theta$ be the permutation $(0 a)(b c)$. Let $\left\{\left(i, j_{s}, \sigma_{s}\right)\right\}_{s=0}^{3}$ be the four face pairings with $i$ as the first element. Here $\sigma_{s}$ glues the $s^{\text {th }}$ face of $\tau_{i}$ to some face of $\tau_{j_{s}}$. Note that $\left\{\left(j_{s}, i, \sigma_{s}^{-1}\right)\right\}_{s=0}^{3}$ are also face pairings in $T$.

Define a new triangulation $T^{\prime}$ by crushing the tetrahedron $\tau_{i}$ along the $a^{\text {th }}$ quad, as follows: Delete $\tau_{i}$ from $T$. Delete all of the face pairings $\left\{\left(i, j_{s}, \sigma_{s}\right)\right\}_{s=0}^{3}$. Replace the face pairing $\left(j_{s}, i, \sigma_{s}^{-1}\right)\left(\right.$ if $\left.i \neq j_{s}\right)$ with

$$
\left(j_{s}, j_{\theta(s)}, \sigma_{\theta(s)} \cdot R_{(s, \theta(s))} \cdot \sigma_{s}^{-1}\right),
$$

for $s \in\{0,1,2,3\}$. Here $R_{(s, \theta(s))}$ is the rotation of the model tetrahedron, about the edge with vertices $\{0,1,2,3\}-\{s, \theta(s)\}$, which takes face $s$ to face $\theta(s)$. Finally, no face of any model tetrahedron in $T^{\prime}$ is glued to itself - thus $T^{\prime}$ is a triangulation.

To keep track of this operation it may help to refer to the picture of a quad of type 3 shown on the right hand side of Figure 2.

Now suppose that $p$ is a polarization of the triangulation $T$; that is, $p$ is a map from the set of tetrahedra to the set $\{0,1,2,3\}$. Produce a new triangulation $T^{\prime}$ by crushing $T$ along $p$ : To begin with let $T^{\prime}$ be an exact copy of $T$. Now, for each $i=1,2, \ldots, \operatorname{size}(T)$ do one of two things; If $p\left(\tau_{i}\right)=0$ simply go on to $i+1$. If $p\left(\tau_{i}\right) \neq 0$ then remove $\tau_{i}$ by crushing along the $p\left(\tau_{i}\right)$ quad, as above, and go on to $i+1$.

We now have:

Theorem 13.1. There is a polynomial-time algorithm that, given a triangulation $T$ and a polarization $p$, produces $T^{\prime}$, the triangulation of $T$ crushed along $p$.

Crushing $T$ along the polarization determined by a non-vertex-linking normal surface $S$ will be called crushing $T$ along $S$.

Theorem 13.2. Suppose $T$ is a triangulation so that the connect sum $\#|T|$ is a homology three-sphere. Suppose $S$ is a non-vertex-linking normal two-sphere. Then the triangulation $T^{\prime}$, obtained by crushing $T$ along $S$, satisfies $\#\left|T^{\prime}\right| \cong \#|T|$.

Proof. Theorem 5.9 of Jaco and Rubinstein's paper [16] essentially claims this result for any closed, orientable three-manifold $|T|$ with the caveat that some connect summands of $|T|$ homeomorphic to lens spaces may by omitted from the crushed $\left|T^{\prime}\right|$. See also [3, Theorem 3.1].

However, by Lemma 3.6 no non-trivial lens space appears as a connect summand of the homology three-sphere $|T|$. Finally, omitting $S^{3}$ summands does not change the connect sum. The result follows. 


\section{Rubinstein And Thompson's Theorem}

We use Casson's version [5] of the proof of Theorem 1.1. Chapter 6 of [3] gives a more detailed exposition of Casson's algorithm.

Theorem 14.1 (Casson [5]). The three-sphere recognition problem lies in PSPACE; there is a polynomial-space algorithm that, given a triangulation $T$, decides whether or not $|T|$ is homeomorphic to the three-sphere.

Proof. We give only a sketch of Casson's version of the RubinsteinThompson algorithm. Begin with a triangulation $T_{0}=T$. Check, using Theorems 3.4 and 3.5 , that $T_{0}$ is a homology three-sphere. Inductively we have a triangulation $T_{i}$.

If $T_{i}$ is not zero-efficient then apply Lemma 4.13 to find $S_{i} \subset\left|T_{i}\right|$, a fundamental non-vertex-linking normal two-sphere. Let $T_{i+1}$ be the triangulation obtained by crushing along $S_{i}$. This requires Theorem 13.1.

If $T_{i}$ is zero-efficient use Lemma 4.13 to search for almost normal two-spheres. If some component of $T_{i}$ does not contain an almost normal two-sphere then by Theorem 13.2 and Theorem 4.12 the manifold $|T|$ was not the three-sphere. If $S_{i}$ is an almost normal two-sphere inside a component $T^{\prime}$ of $T_{i}$ then let $T_{i+1}=T_{i}-T^{\prime}$.

This completes the description of Casson's algorithm. If $T_{n}$ is nonempty, then $|T|$ was not the three-sphere. If $T_{n}$ is empty then $|T|$ was homeomorphic to the three-sphere. Both of these again use Theorem 13.2 .

Note that $\operatorname{size}\left(T_{i}\right)+i \leq \operatorname{size}(T)$ as either crushing along a polarization or deleting a component always reduces the number of tetrahedra by at least one. This completes the sketch.

\section{Showing the PRoblem Lies in NP}

We are now in a position to prove:

Theorem 15.1. The three-sphere recognition problem lies in NP.

Proof. Suppose that $T$ is a triangulation of the three-sphere. The certificate is a sequence of pairs $\left(T_{i}, v\left(S_{i}\right)\right)$ with the following properties.

- $T=T_{0}$.

- $S_{i}$ is a normal or almost normal two-sphere, contained in $\left|T_{i}\right|$, with weight $\left(S_{i}\right) \leq \exp \left(\operatorname{size}\left(T_{i}\right)\right)$.

- If $S_{i}$ is normal then $S_{i}$ is not vertex linking and $T_{i+1}$ is obtained from $T_{i}$ by crushing along $S_{i}$.

- if $S_{i}$ is almost normal then $S_{i}$ normalizes to vertex linking twospheres (or the empty set), in both directions. Also, $T_{i+1}$ is 
obtained from $T_{i}$ by deleting the component $T^{\prime}$ of $T_{i}$ which contains $S_{i}$.

- Finally, the last triangulation $T_{n}$ is empty, as is $S_{n}$.

Note that existence of the certificate is given by our proof of Theorem 14.1. The only task remaining is to check the certificate. There are two subtle points - we do not verify that the $S_{i}$ are fundamental nor do we check that the $T_{i}$ containing almost normal two-spheres are zero-efficient.

Instead, since the $S_{i}$ are fundamental, they obey the weight bounds given in Lemma 4.8; that is, weight $\left(S_{i}\right) \leq \exp \left(\operatorname{size}\left(T_{i}\right)\right)$.

Suppose a certificate $\left(T_{i}, v\left(S_{i}\right)\right)$ is given as above, for the triangulation $T$. Check, using Theorems 3.4 and 3.5, that $T$ is a triangulation of a homology three-sphere.

By Lemma 3.1 check that $T=T_{0}$. Using Theorem 4.6 verify that $S_{i}$ is a connected normal or almost normal surface. Using Lemma 4.5 compute the Euler characteristic of $S_{i}$. (Here we are using the fact that weight $\left(S_{i}\right) \leq \exp \left(\operatorname{size}\left(T_{i}\right)\right)$ in order to compute Euler characteristic in time polynomial in size $\left(T_{i}\right)$.) This verifies that $S_{i}$ is a two-sphere.

If $S_{i}$ is normal, by Theorem 13.1, crush $T_{i}$ along $S_{i}$ in time at most polynomial in size $\left(T_{i}\right)$. Then check, using Lemma 3.1, that $T_{i+1}$ agrees with the triangulation obtained by crushing $T_{i}$.

If $S_{i}$ is almost normal, we need to check that $T^{\prime}$, the component of $T_{i}$ containing $S_{i}$, has $\left|T^{\prime}\right| \cong S^{3}$. Using Theorem 12.1 normalize $S_{i}$ in both directions in time at most polynomial in size $\left(T_{i}\right)$. If all components of the two normalizations norm $\left(S_{i}^{+}\right)$and norm $\left(S_{i}^{-}\right)$are vertex linking twospheres then $T^{\prime}$ is a triangulation of the three-sphere, by Theorem 10.3. Finally, use Lemma 3.1 to check that the triangulation $T_{i}-T^{\prime}$ is identical to $T_{i+1}$.

\section{REFERENCES}

[1] Ian Agol. Personal communication. [3]

[2] Ian Agol, Joel Hass, and William Thurston. 3-manifold knot genus is NPcomplete. In Proceedings of the Thirty-Fourth Annual ACM Symposium on Theory of Computing, pages 761-766 (electronic), New York, 2002. ACM. arXiv:math/0205057. [1, 3, 11, 15]

[3] Alexandre Barchechat. Minimal triangulations of 3-manifolds. PhD thesis, U.C. Davis, 2003. arXiv:math/0307302. [2, 3, 8, 13, 16, 34, 35, 36]

[4] Benjamin A. Burton. Regina: Normal surface and 3-manifold topology software. http://regina.sourceforge.net/, 1999-2009. [3]

[5] Andrew J. Casson. The three-sphere recognition algorithm, 1997. Lecture at MSRI, USA. [1, 3, 4, 13, 34, 36]

[6] Andrew J. Casson and Cameron McA. Gordon. Reducing Heegaard splittings. Topology Appl., 27(3):275-283, 1987. [16] 
[7] Bruce Randall Donald and David Renpan Chang. On the complexity of computing the homology type of a triangulation. In 32nd Annual Symposium on Foundations of Computer Science (San Juan, PR, 1991), pages 650-661. IEEE Comput. Soc. Press, Los Alamitos, CA, 1991. http://citeseer.ist.psu.edu/chang94computing.html. [8]

[8] David Gabai. Foliations and the topology of 3-manifolds. III. J. Differential Geom., 26(3):479-536, 1987. [1, 3]

[9] Michael R. Garey and David S. Johnson. Computers and intractability. W. H. Freeman and Co., San Francisco, Calif., 1979. A guide to the theory of NP-completeness, A Series of Books in the Mathematical Sciences. [5]

[10] Joel Hass, Jeffrey C. Lagarias, and Nicholas Pippenger. The computational complexity of knot and link problems. J. ACM, 46(2):185-211, 1999. arXiv:math/9807016. $[3,4,7,8,12,13]$

[11] Alan Hatcher. Notes on basic 3-manifold topology. 2001. http://www.math.cornell.edu/ hatcher/3M/3Mdownloads.html. $[2,7]$

[12] Costas S. Iliopoulos. Worst-case complexity bounds on algorithms for computing the canonical structure of finite abelian groups and the Hermite and Smith normal forms of an integer matrix. SIAM J. Comput., 18(4):658-669, 1989. [8]

[13] S. V. Ivanov. Recognizing the 3-sphere. Illinois J. Math., 45(4):1073-1117, 2001. http://www.math.uiuc.edu/ hildebr/ijm/winter01/final/ivanov.html. [3]

[14] S. V. Ivanov. The computational complexity of basic decision problems in 3-dimensional topology. Geom. Dedicata, (131):1-26, 2008. [3]

[15] William Jaco and Ulrich Oertel. An algorithm to decide if a 3-manifold is a Haken manifold. Topology, 23(2):195-209, 1984. [3]

[16] William Jaco and J. Hyam Rubinstein. 0-efficient triangulations of 3-manifolds. J. Differential Geom., 65(1):61-168, 2003. arXiv:math/0207158. [2, 8, 10, 12, $13,16,34,35]$

[17] William Jaco and Jeffrey L. Tollefson. Algorithms for the complete decomposition of a closed 3-manifold. Illinois J. Math., 39(3):358-406, 1995. [7, 11, $13]$

[18] Simon A. King. The size of triangulations supporting a given link. Geom. Topol., 5:369-398 (electronic), 2001. arXiv:math/0007032. [16]

[19] S. V. Matveev. Algorithms for the recognition of the three-dimensional sphere (after A. Thompson). Mat. Sb., 186(5):69-84, 1995. [3]

[20] Sergei Matveev. Algorithmic topology and classification of 3-manifolds, volume 9 of Algorithms and Computation in Mathematics. Springer-Verlag, Berlin, 2003. $[3,7]$

[21] Christos H. Papadimitriou. Computational complexity. Addison-Wesley Publishing Company, Reading, MA, 1994. [5]

[22] J. Hyam Rubinstein. Polyhedral minimal surfaces, Heegaard splittings and decision problems for 3-dimensional manifolds. In Geometric topology (Athens, GA, 1993), pages 1-20. Amer. Math. Soc., Providence, RI, 1997. [3, 9]

[23] Joachim H. Rubinstein. The solution to the recognition problem for $S^{3}, 1992$. Lectures in Haifa, Israel. [1, 3, 12]

[24] Joachim H. Rubinstein. An algorithm to recognize the 3-sphere. In Proceedings of the International Congress of Mathematicians, Vol. 1, 2 (Zürich, 1994), pages 601-611, Basel, 1995. Birkhäuser. [3] 
[25] Saul Schleimer. Almost normal Heegaard splittings. PhD thesis, U.C. Berkeley, 2001. http://warwick.ac.uk/ masgar/Maths/thesis.pdf. [2, 3, 16]

[26] Abigail Thompson. Thin position and the recognition problem for $S^{3}$. Math. Res. Lett., 1(5):613-630, 1994. [1, 3, 12]

Department of Mathematics, University of Warwick, Coventry, CV4 $7 \mathrm{AL}, \mathrm{UK}$

E-mail address: s.schleimer@warwick.ac.uk 